This is a post-peer-review, pre-copyedit version of an article published in Theory and Decision. The final authenticated version is available online at: https://doi.org/10.1007/s11238-020-09779-9.

\title{
On the aversion to incomplete preferences*
}

\author{
Ritxar Arlegi ${ }^{1 \dagger}$, Sacha Bourgeois-Gironde ${ }^{2}$, Mikel Hualde ${ }^{1}$ \\ ${ }^{1}$ Department of economics, Public University of Navarre and Institute for Advanced Research in \\ Business and Economics (INARBE), Campus Arrosadia s/n - 31006 Pamplona, Navarra, Spain. \\ ${ }^{2}$ Laboratoire d'economie mathematique et de microeconomie appliquee (LEMMA), \\ Universite Paris 2 Pantheon-Assas 12 place du Pantheon, 75005 Paris.
}

June 25, 2020

\begin{abstract}
We propose an axiomatization of aversion to incomplete preferences. Some prevailing models of incomplete preferences rely on the hypothesis that incompleteness is temporary and that by keeping their opportunity set open individuals reveal a preference for flexibility. We consider that the maintenance of incomplete preference is also aversive. Our model allows us to show how incompleteness induces an aversive attitude in two different ways: intrinsic and instrumental. Intrinsic aversion holds when one instance of incomplete preference in the set suffices to decrease its utility. Instrumental aversion holds only insofar dominating options are affected by incompleteness. Given two partially overlapping sets of axioms on the binary relation over sets we formalize their consistency with the two types of aversion to incompleteness. Finally, we relate our model to the classical Sen's distinction between tentative and assertive incompleteness. The spelling out of this distinction in the terms of our approach uncovers to what extent aversion to incompleteness may be compatible with preference for flexibility.
\end{abstract}

JEL Classification: D01, D91

Keywords: Aversion to incomplete preferences. Preference for flexibility. Ranking sets.

*Financial support from the Spanish Ministry of Science and Technology (Project ECO2015-65031-R MINECO/FEDER, UE) and the Public University of Navarre is acknowledged.

${ }^{\dagger}$ Corresponding author: e-mail: rarlegi@unavarra.es 


\section{Introduction}

Incompleteness of preferences may relate to different sources: objective or subjective, i.e. either pointing to incomparable features among the alternatives themselves, or to an epistemic state of decision uncertainty of the individual (for example, due to lack of information). In the former case incompleteness is, in principle, unsolvable. In the latter case, elucidating what the expectations of the individual about the solvability of incompleteness are turns to be crucial in order to evaluate its welfare implications.

The interpretation and resolution of incompleteness in terms of preference for flexibility (Kreps (1979), Arlegi and Nieto (2001a), Danan and Ziegelmeyer (2006)) and some choicedeferral models (Gerasimou (2017)) indicate that the individual is temporally uncertain about her best choice but expects for preferences to be resolved at a later point in time when the final choice has to be made. In this case postponing the decision generates an instrumental value. It may be the case, on the contrary, that incomparability is not expected to be solved when the final choice is due. This may be because alternatives are objectively incomparable or because, being subjectively incomparable, the individual does not expect to elucidate incomparabilities at time. In this case, a lucid individual will not presume that deferred choice will be useful. Even more, the individual might be psychologically averse to such indecisiveness and to the presence in the opportunity set of incomparable alternatives. In that case we are led to model a phenomenon that is the opposite of preference for flexibility, and which we label as "aversion to incomplete preferences".

In this article, we axiomatically characterize how incompleteness induces, in different ways, an aversive attitude. The focus of our model is the potential psychological discomfort incurred by an individual if an option creating some preference incompleteness is added to a set. Following Sen's terminology (Sen (1988, 1997), Pattanaik and Xu (1990)), and unlike him, our model does not admit any intrinsic value of freedom of choice but we accept that it has instrumental value when an option added to a set increases its indirect utility (the utility of the best alternative in the set) and does not generate any aversion due to incompleteness. This is therefore a different point than granting a positive value to a preference for flexibility. The latter can be the case when the individual knows that her uncertain preference can be solved later, but not when her preference relation is definitely incomplete. There is then no added value by choice deferral and no preference for flexibility.

We envision two types of situations, each captured by a same basic model and supported by partially overlapping sets of axioms. We first model an intrinsic aversion to incomplete- 
ness, corresponding to a situation in which an alternative is added to the option set and potentially creates some incomplete preference that we assume is psychologically costly. The presence of that alternative in the option set intrinsically worsens its value. It is a different point than the one discussed in Sonsino and Mandelbaum (2001) according to which increasing a set cardinality creates a trade-off between added value due to greater flexibility of choice and decreased value due to complexity aversion. We do not grant positive value to flexibility of choice and complexity is aversive only with respect to incomplete preferences, that is, when the higher cardinality of a set increases the extent of incompleteness in the set.

In that first situation, we assume that the individual compares all the alternatives present in her option set. Facing a pair that cannot be compared generates some psychological cost which is not solved by choice-deferral. With respect to a second type of situation we model an instrumental aversion to incompleteness. In that situation an incomplete preference induced by the addition of an alternative in the option set has a psychological cost only if it involves some undominated options. It means that induced incompleteness by enlargement of the option set does not affect the welfare of the individual when it involves irrelevant options.

Let us imagine a vegetarian individual presented with the menu \{vegetarian meal; chicken $\}$ and in another occasion with the menu \{vegetarian meal; chicken; turkey\}. We can presume that the preference of this individual is incomplete between chicken and turkey and that she is not interested anyway, in standard circumstances, in making comparisons between the two fowl dishes. The incomplete preference between them does not induce any kind of aversion as no choice will have to be borne onto them. But if the individual is facing the addition of another vegetarian meal that she cannot compare with the one already present, she experiences incompleteness only for the dominating part of the menu, i.e. the options among which she is inclined to choose. ${ }^{1}$

From a methodological point of view, our approach connects with a tradition of works where the preference for flexibility and the value of freedom of choice are studied by means of the axiomatic characterization of set ranking rules that are "extensions" of an underlying binary relation of preferences over the single options (see for example Bossert et al. (1994), Puppe (1996) or Arlegi and Nieto (2001a)). In those models agents always display a preference for enlarged menus whereas we study the opposite phenomenon, namely, the

\footnotetext{
${ }^{1}$ In section 3 we develop a more detailed discussion about decisional contexts where either the intrinsic or the instrumental approach are more suitable.
} 
possibility for the decision maker to prefer smaller sets as a consequence of the aversion to incompleteness in preferences.

The preference for restricting one's own opportunity set has been checked both experimentally and empirically in different works (see Iyengar and Lepper (2000) and Sonsino and Mandelbaum (2001)). Also, different theoretical models exist to explain it as a result of different hypotheses.

Gul and Pesendorfer (2001), Dekel et al. (2007) and Sarver (2008) interpret preference for smaller sets as a preference for commitment in the presence of regret and temptation aversion. In these and related models, alternatives are fully specified as lotteries so that they are perfectly comparable in expected utility terms. Then, the preference for smaller sets comes from the fear of having chosen a wrong alternative. This is usually modeled by making the utility of the lotteries state contingent. That is, the value of the lottery depends on an exogenous state of nature.

As already explained, two crucial distinctive aspects of our model are, on the one hand, that some alternatives are incomparable, and on the other hand, that incomparabilities are not solved at the moment of the final choice. Thus, we assume that the decision maker is endowed with a unique, well-defined but incomplete binary relation $P$, defined over the single alternatives. Alternatives are abstract objects which do not need to be bestowed with any additional structure; some alternatives are incomparable, and the individual is averse to the presence of incomparable alternatives. This sort of aversion can itself have different psychological sources. It could be a matter of simple "noise aversion", or it could be associated to the psychological connotations of the very act of choice, such as the sense of responsibility: Very likely, in "Sophie's choice" novel, Sophie would have preferred not to be forced to choose which of her children deserved living, a preference that is hardly interpretable in terms of regret or noise aversion. ${ }^{2}$ Another conceptual difference of our approach is that, given that in our model incomparabilities prevail when the final choice has to be made, there is no way to know whether the final choice is "right" or "wrong".

These conceptual differences lead also to basic formal divergences with respect to regret and temptation models. For example, in our model there is no room for a preference chain such as $\{x\} \succ\{x, y\} \succ\{y\}$ or $\{x\} \succ\{x, y\} \sim\{y\},{ }^{3}$ which is especially plausible in regret

\footnotetext{
${ }^{2}$ In "Sophie's choice" Styron (1979), Sophie is an Auschwitz prisoner who is obliged to choose which one of her two children would be gassed.

${ }^{3} \succ$ denotes the strict preference over sets and $\sim$ the indifference. We define these binary relations formally in Section 2 .
} 
and temptation theories. ${ }^{4}$ In temptation models (e.g. Gul and Pesendorfer (2001)) a commitment ranking and a temptation ranking over the alternatives coexist. The commitment ranking represents the ranking of the alternatives when no other choice is available. The temptation ranking represents the agent's desires when the final choice has to be made. When $y$ is tempting and the agent anticipates a self-control effort in order to choose $x$ when $y$ is available, then, at the first stage of the decision process, $\{x\} \succ\{x, y\}$ (the agent prefers to avoid facing the temptation of choosing $y$, which is dominated by the commitment ranking) and $\{x, y\} \succ\{y\}$ (the individual anticipates the choice of $x$ over $y$ ). In regret models (e.g. Sarver (2008)) $x$ is preferred to $y$ in terms of expected utility, and the individual prefers $\{x\}$ to $\{x, y\}$ in order to avoid the feeling of regret if $x$ results to be ex post inferior to $y$. At the same time, the individual prefers having the opportunity to choose $x$ (set $\{x, y\})$ rather than being forced to choose the ex ante inferior alternative $y$. Instead, in our model, the incompleteness of preferences during the considered span of time does not allow to establish any kind of (ex ante or ex post) preference between $x$ and $y$. Thus, $\{x\} \succ\{x, y\}$ reveals an incomparability between $x$ and $y$ to which the individual is averse and, by the symmetry of this incomparability, $\{y\} \succ\{x, y\}$ as well.

Ortoleva (2013) explains the preference for smaller sets in terms of "thinking aversion". His proposal is also constructed on the basis of the final choice at the second stage of the process and an anticipation of the necessary cost of thinking to discern which is the best alternative for such final choice. As already noted, in our model incomparabilities are unsolvable and remain unsolved at the time when the final choice has to be made. This could be because incomparabilities are not solvable by "thinking" under any cost of computation (for example, if the alternatives are intrinsically incomparable) or because the agent is aware that she has not the computational capacities to solve them satisfactorily when the final choice has to be made (for example because she knows that at the final stage the necessary information to do so will not be available).

Similarly to Guerdjikova and Zimper (2008) and Danan et al. (2012), we consider a model where the individual may be uncertain about her preference even at the moment of the final choice and she is averse to indecisiveness. This idea is very close to our idea of aversion to incomplete preferences. Danan et al. (2012) propose a rule to rank sets of state-dependent utility lotteries which, in fact, and under the appropriate reformulation, is a particular member of a more general family of binary relations over sets that we characterize

\footnotetext{
${ }^{4}$ This preference chain would is a possibility under the Set Betweenness axiom (Gul and Pesendorfer (2001)): $A \succsim B$ implies $A \succsim A \cup B \succsim B$, which is distinctive of these models.
} 
in Section 4 and call " $P$-instrumental consistent".

In section 2 we present our general model of aversion to incomplete preferences. An incomplete preference relation is defined relatively to a strict indubitable relation, $P$. In section 3 we define formally and discuss the two types of aversion to incompleteness which we model, intrinsic vs. instrumental, in relation with $P$. In section 4.1 we present the set of axioms that can give sense to an aversion induced by a set-enlargement potentially generating incompleteness, and characterize axiomatically the two types of aversion to incomplete preferences. Section 4.2 discusses the results, noticing that the binary relation over sets needs not to be transitive in order to display an aversion to incomplete preferences.

All the related works mentioned above propose alternative utility representations for the preferences over sets and therefore specify complete rankings over them. The rules that we characterize are, in general, incomplete and may be intransitive. In fact, we take as natural that the trade-off between incomparabilities in the set and the quality of its alternatives leads to intransitivities when comparing different sets. Then, it is important to know whether we can infer transitive and/or complete rankings that are consistent with our basic relation $P$ on the basis of our alternative definitions. In section 4.3 we study the formal consequences of assuming transitivity of the binary relation over sets, showing some corollaries and examples.

Finally, we feel the need to clarify how our proposal stands with respect to the more usual approach to incomplete preferences in terms of preference for flexibility. We substantially depart from the usual notion of preference for flexibility. In most models of preference for flexibility, either axiomatic (Kreps (1979), Puppe (1996), Nehring (1999), Pejsachowicz and Toussaert (2017)) or in view to provide a choice-theoretical basis to elicit incomplete preferences (Danan and Ziegelmeyer (2006)), the individual may be temporally uncertain about her preferences and expect them to be solved at a later point. Due to this mental state, the preservation of a larger set that contains an incomplete preference holds instrumental value. In our approach the introduction of incompleteness is always costly. But, essentially, the individual is not uncertain about her, albeit incomplete, preferences and the definite incomparability of the concerned alternatives. The notion of aversion to incompleteness and of preference for flexibility being thus orthogonalized, we can think of ways the two phenomena can be combined. We develop in section 5 a model in which the two phenomena can coexist, in contrast with an impossibility result shown in Pejsachowicz and Toussaert (2017). Guerdjikova and Zimper (2008) also combine the aversion to indecisiveness phenomenon of preferring smaller sets with the preference for flexibility effect. Their 
conception of aversion to indecisiveness is very close to our notion of intrinsic aversion to incompleteness, but their conception of preference for flexibility is not related with the uncertainty about the future preferences (like we assume, following Kreps (1979)), but on the fact that at the second stage nature restricts the availability of opportunities in the sets. At this point our model and results depart from theirs.

To conclude, Section 6 (Appendix) provides the proofs of the theorems and Corollaries 3 and 4. The proofs of Propositions 1 to 4 (independence of the axioms) are provided in the Online Appendix.

\section{Basic notation}

$X$ denotes a finite set of opportunities. $\pi(X)$ denotes the set of all nonempty subsets of $X$. $P$ denotes an asymmetric binary relation defined on $X$ interpreted as an indubitable strict preference. Sometimes, when for a pair of alternatives $x, y \in X, x P y$ we will say that $x$ dominates $y . \otimes$ is defined by: $\forall x, y \in X, x \otimes y$ if $\neg(x P y)$ and $\neg(y P x) . x \otimes y$ is therefore interpreted as incomparability or absence of preference between $x$ and $y$. Note that $\otimes$ is, by definition, a symmetric binary relation.

We assume that the presence of incomparability in an opportunity set involves a psychological cost to which the individual is averse. We investigate elicitation-like results, in particular, we are interested in the axiomatic characterization of preference over sets that are interpretable as if there were an underlying preference $P$ over the alternatives with which such preference over sets is consistent under the assumption that the individual is averse to $P$-incomparability. With that purpose, $\succsim$ denotes a binary relation defined on $\pi(X)$, whose interpretation is that of a weak preference over sets. $\succ$ and $\sim$ denote, respectively, the asymmetric and symmetric factors of $\succsim$. It is important to remark that the usage of the

term "preference" to refer to either $P$ or $\succsim$ does not preclude that they are transitive and/or complete binary relations. In fact, as it will be seen, they are not assumed, in general, to satisfy transitivity or completeness. 


\section{P-consistency in terms of the cost of incomplete prefer- ences}

\subsection{Definitions}

We present two alternative definitions of $P$-consistency with aversion to incomplete preferences. We later discuss the conceptual differences and characterize the two notions axiomatically.

Definition 1. Let $P$ be an asymmetric binary relation defined on $X$. A reflexive binary relation $\succsim \subseteq \pi(X) \times \pi(X)$ is $P$-consistent with the intrinsic aversion to incomplete preferences (henceforth "P-intrinsic consistent") if it satisfies the following condition:

$$
\forall A \in \pi(X), \forall x \in X \backslash A\left\{\begin{array}{c}
A \cup\{x\} \succ A \text { if } x P a \text { for all } a \in A \\
A \succ A \cup\{x\} \text { if } \exists a \in A \text { such that } x \otimes a \\
A \cup\{x\} \sim A \text { otherwise }
\end{array}\right.
$$

For the next definition let us consider, for any $A \in \pi(X), \max _{P}(A)=\left\{a \in A: \nexists a^{\prime} \in A\right.$ such that $\left.a^{\prime} P a\right\}$ :

Definition 2. Let $P$ be an asymmetric and transitive binary relation defined on $X . A$ reflexive binary relation $\succsim \subseteq \pi(X) \times \pi(X)$ is $P$-consistent with the instrumental aversion to incomplete preferences (henceforth " $P$-instrumental consistent") if it satisfies the following condition:

$$
\forall A \in \pi(X), \forall x \in X \backslash A\left\{\begin{array}{c}
A \cup\{x\} \sim A \text { if } \exists a \in A \text { such that aPx } \\
A \cup\{x\} \succ A \text { if } \exists a \in \max _{P}(A) \text { such that } x P a \\
A \succ A \cup\{x\} \text { otherwise }
\end{array}\right.
$$

In both cases $P$-consistency is defined on the basis of requirements on the elementary question about how the addition a new opportunity, $x$, affects the value of a given set.

$P$-intrinsic consistency (Definition 1 ) states that $x$ improves a set if it $P$-dominates all the alternatives in the set, $x$ worsens the set if it is incomparable with some alternative in 
the set (thus inducing some aversion to incompleteness), and finally $x$ does not affect the value of the set otherwise, that is, if it is related by means of $P$ with all the alternative in the set, but in such a way that at least one of the alternatives $P$-dominates $x$.

According to $P$-instrumental consistency (Definition 2), for a new alternative, $x$, not to affect the set it is sufficient that there exists some alternative in the set that dominates it. For $x$ to worsen the set it is necessary that it is incomparable with all the alternatives in the $P$-maximal subset, and not with just one alternative in the set, as it is the case of the intrinsic approach. Finally, for $x$ to improve the set it should dominate some alternative in the $P$-maximal subset.

According to Definition 1 the individual is averse to the mere presence of incompleteness among the alternatives in the set. According to Definition 2 incompleteness is only harmful when affecting non dominated alternatives. To better illustrate the difference, imagine three alternatives $a, b, c$ such that $a P b ; a P c$ and $c \otimes b$. Under Definition 1 we have $\{a, b\} \succ\{a, b, c\}$ even though $c$ is dominated. Under Definition 2 we have $\{a, b\} \sim\{a, b, c\}$.

There are decisional contexts where $P$-intrinsic consistency fits better and others where the "instrumental" approach seems to be more appropriate. The following four factors may be relevant as to the suitability of one or other of the approaches:

(i) Whether the final output of the choice process consists of selecting a single alternative or ranking the elements in the sets (or a subset of them). In the former case, the instrumental approach makes more sense: dominated alternatives will never be chosen so that incomparabilities among those alternatives are innocuous. In the later case (for example, selecting and ranking a group of candidates to cover a set of positions), the outcome of the choice process may contain dominated alternatives and therefore aversion to incompleteness may interfere even if incomparabilities hold among dominated alternatives.

(ii) The "strength" of the value judgment attached to $P$. In some contexts $P$ can be interpreted as an absolutely indubitable preference, so that it is evident that dominated alternatives are totally irrelevant and so are the incomparabilities among them. In other situations $P$ could be associated to a milder value judgment. Recall our legendary vegetarian. The addition of the turkey meal to the \{Vegetarian meal, chicken\} menu did not involve any aversion. Imagine now that the person is not vegetarian, but is simply in the mood of eating vegetables that day. Again she is proposed first the menu \{Vegetarian meal, chicken\} and later the menu \{Vegetarian meal, chicken, pork\} and again she is not sure of whether she prefers pork or chicken. She is just in the mood of a vegetarian meal but the addition of the pork option may involve an undesired "noise" in her decision problem. 
(iii) The overall size of the decision problem and the nature of $P$. Under the intrinsic approach an alternative $x$ worsens $A$ if, for example, $x P a$ for all $a \in A \backslash a^{\prime}$ and $x \otimes a^{\prime}$. This might be a counterintuitive result, especially when $A$ contains a large number of alternatives. ${ }^{5}$ However, in situations where a few alternatives are involved in the decision problem and the decision maker is aware that her preferences are "messy" (notice that in the intrinsic approach $P$ may even contain cycles) it might make sense that, in the example, the additional load of incomparability of $x$ is not able to overcome the confidence that may provide the fact that $x$ is better than a few existing alternatives.

(iv) The kind of computational abilities predicated on the agent. Under the intrinsic approach, in order to determine the effect of adding a new alternative to a set whose cardinality is $n$, the agent should be able to make the $n$ pairwise comparisons. Under the instrumental approach, in order to make a definite judgement about the effect of adding any possible alternative, she should be able to identify the maximal $P$-subset as a previous step. This could be considered a more sophisticated task so that, as long as it is assumed that performing it is an issue, the intrinsic approach could be considered more suitable.

Our choice of the terms "intrinsic" and "instrumental" is not gratuitous. There are some analogies with Sen (1988) distinction between the intrinsic and the instrumental values of freedom of choice. His distinction, like ours, is determinant for the evaluation of opportunity sets. According to Sen, the availability of more opportunities (freedom of choice) may have an instrumental value, as a means to other ends. The most evident example is the indirect utility criterion of the standard consumer's theory, where the addition of a new opportunity is only valuable as long as it is not dominated by another one in the set. But freedom of choice may also have intrinsic importance, so that the mere possibility to choose is valuable as an end of its own right. Analogously, in our case, aversion to incompleteness may have an intrinsic nature (the mere presence of incompleteness in the opportunity set is psychologically costly) or an instrumental one (incompleteness is irrelevant when affecting dominated opportunities).

\footnotetext{
${ }^{5}$ We thank an anonymous referee for proposing this example to address the limits in the scope of the intrinsic approach.
} 


\section{Characterization results}

\subsection{Axiomatic characterizations}

Next we present and discuss several axioms in order to explore the axiomatic structure of the intrinsic and instrumental aversions to incompleteness.

- Revealed Indifference (RI)

$\forall x, y \in X,\{x, y\} \succ\{y\} \Leftrightarrow\{x, y\} \sim\{x\}$

RI states that the fact that adding $x$ to $y$ involves an improvement reveals that adding $y$ to $x$ would make the set remain indifferent, and vice versa. RI is a simple axiom, but crucial in our model. It is in line with the classical indirect utility criterion in the "if" direction $(\{x, y\} \succ\{y\}$ implies $\{x, y\} \sim\{x\})$ but not in the reverse one. In this sense, the axiom is implicitly assuming that there are no absolute certain indifferences: Under such type of indifferences, if $x$ is undoubtedly indifferent to $y$, it makes sense to assume that both $\{x, y\} \sim\{x\}$ and $\{x, y\} \sim\{y\}$ hold . This is to say that our model applies to decisional contexts where the absence of preference involves some component of dilemma. The decision is sufficiently important, so that the individual feels pressed to make the best choice rather than leave it random. ${ }^{6}$ Recalling Sen (1997) and his two possible interpretations of Buridan's ass problem, "the less interesting, but more common interpretation is that the ass was indifferent between the two haystacks (...). The second-more interesting-interpretation is that the ass could not rank the two haystacks and had an incomplete preference over this pair". 7

- Simple comparability (SC)

$\forall x, y \in X, \neg(\{x, y\} \succsim\{x\})$ implies $\{x\} \succ\{x, y\}$.

SC states that any twofold set is comparable with any of its nonempty subsets, that is, the decision maker is always capable to tell whether adding an alternative to a singleton involves an improvement, worsening, or leaves the situation indifferent.

\footnotetext{
${ }^{6}$ Schwartz et al. (2002) also distinguish between contexts where decisions are or not meaningful in order to claim that individuals would act as either satisficers or maximizers.

${ }^{7}$ In Section 5, under the perspective of the different axiomatic characterization results, we include a discussion about the possibility of including both interpretations of the absence of preference under a unique model.
} 
- Monotonicity Consistency (MC)

$\forall A \in \pi(X), \forall x \in X \backslash A,\{x, a\} \succ\{a\} \forall a \in A \Rightarrow A \cup\{x\} \succ A$

MC requires that if $x$ improves all the singletons compounding a set, then it should have the same effect on the set itself. Therefore, it expresses some kind of separability in the corresponding effect.

- Indifference Absorption (IA)

$$
\begin{aligned}
& \forall A \in \pi(X), \forall x \in X \backslash A,\{x, a\} \succsim\{a\} \forall a \in A \text { and } \exists a^{*} \in A \text { s.t }\left\{x, a^{*}\right\} \sim\left\{a^{*}\right\} \\
& \Rightarrow A \cup\{x\} \sim A
\end{aligned}
$$

IA states that, if $x$ does not worsen any of the singletons of the set and there exists at least one singleton $\left\{a^{*}\right\}$ such that $x$ does not either improve or worsen the situation when added to it, then the addition of $x$ to the whole set should not affect it. he intuition behind this axiom is that the presence of $a^{*}$ absorbs the irrelevance of $x$ even though $x$ might be better than other alternatives in the set.

- Cost Expansion (CE)

$$
\forall A, B \in \pi(X) \text { such that } A \subseteq B, \forall x \in X \backslash B, A \succ A \cup\{x\} \text { implies } B \succ B \cup\{x\}
$$

$\mathrm{CE}$ is coherent with the intuition that incomparability entails some kind of psychological cost which, naturally, perdures when the set is enlarged.

The previous axioms characterize the set of binary relations over sets that are $P$-intrinsic consistent for some $P$ defined over the basic alternatives:

Theorem 4.1. Let $\succsim$ be a reflexive binary relation defined on $\pi(X)$. There exists an asymmetric binary relation $P$ defined on $X$ such that $\succsim$ is $P$-intrinsic consistent if and only if $\succsim$ satisfies RI, SC, MC, IA and CE. Moreover, $P$ is unique.

Proposition 1. Axioms RI, SC, MC, IA and CE are independent

In order to characterize the set of binary relations over sets that are $P$-instrumental consistent for some $P$ defined over the basic alternatives we now present four additional axioms. 
- Cost Consistency (CC)

$\forall A \in \pi(X), \forall x \in X \backslash A,\{a\} \succ\{a, x\} \forall a \in A \Rightarrow A \succ A \cup\{x\}$

$\mathrm{CC}$ is the counterpart of $\mathrm{MC}$ in relation of the negative effect of the addition of a new alternative. It requires that if $x$ worsens all the singletons compounding a set, then it should have the same effect on the set itself. Notice that CC is a weaker version of CE.

- Indifference Chain (IC)

$\forall x, y \in X, \forall A \in \pi(X)$ such that $y \notin A,\{x, y\} \sim\{x\}$ and $A \cup\{x\} \sim A$ imply $A \cup\{y\} \sim A$

According to IC, the irrelevance of an alternative is chained in the sense that, if the decision maker does not take into account neither $y$ when it is added to $x$, nor $x$ when it is added to $A$, then it should not take into account $y$ when it is added to $A$.

- Monotonicity Robustness (MR)

$\forall A \in \pi(X), \forall x, y \in X \backslash A, A \cup\{x\} \succ A, A \succsim A \cup\{y\}$ and $\neg(\{y\} \sim\{x, y\})$ implies $A \cup\{x, y\} \succ A \cup\{y\}$

MR imposes some kind of robustness to the fact that an alternative, $x$, improves a set $A$ in the sense that, if another alternative, $y$, does not improve $A$, and does not "absorb" the improvement of $x$, then adding both alternatives is preferred to adding just the alternative that does not improve the set.

- Cost Robustness (CR)

$\forall A \in \pi(X), \forall x, y \in X \backslash A, A \succ A \cup\{x\}$ and $A \sim A \cup\{y\}$ implies $A \cup\{y\} \succ A \cup\{x, y\}$

CR states that if an alternative worsens a set and another alternative does not affect it, then adding just the alternative that does not affect the set is preferred to adding both alternatives.

The new axioms, together with some of the axioms used in Theorem 4.1 allow to characterize $P$-instrumental consistency.

Theorem 4.2. Let $\succsim$ be a reflexive binary relation defined on $\pi(X)$. There exists an asymmetric and transitive binary relation $P$ defined on $X$ such that $\succsim$ is $P$-instrumental consistent if and only if $\succsim$ satisfies $R I, S C, C C, I C, M R$ and $C R$. Moreover, $P$ is unique.

Proposition 2. Axioms RI, SC, CC, IC, MR and CR are independent 


\subsection{Discussion}

Notice that in theorems 4.1 and 4.2 nothing but reflexivity is imposed on $\succsim$. That is, $\succsim$ might be incomplete and/or intransitive. Completeness and transitivity are sound principles of a significant normative and practical import in economics. There is, however, starting with May (1954) and Tversky (1969), ample experimental and empirical evidence of the violation of both paradigms. Menus are even more complex objects than single alternatives, which suggests that such violations become even more plausible. Besides this general remark, in our approach $\succsim$ constitutes the primitive, observable element of the model. This model tries to explain such observed behavior (the comparison of menus) "as if" it were consistent with a possibly incomplete underlying binary relation, $P$, defined over the alternatives in terms of aversion to incompleteness. This means that it is not necessary that $\succsim$ is either complete or transitive for eliciting an underlying incomplete binary relation over the alternatives whose incompleteness provokes aversion. Nonetheless, in Subsection 4.3 we explore the consequences of imposing transitivity on $\succsim$.

Notice too that the axioms impose conditions only over pairs of sets $(A, B)$ where $B=$ $A \cup\{x\}$. By this we try to focus on elementary comparisons that allow to isolate the phenomenon to be studied (i.e. the aversion to incompleteness of preferences) from other factors than can introduce trade-off or cross effects.

As for the axiomatic characterizations, axioms SC and RI are common to both Theorem 4.1 and Theorem 4.2. They are core elements of the model and they are related directly to the existence or the non-existence of a relationship between the alternatives by means of $P$. In particular, in the proofs of the theorems, RI and SC allow to recover the unique binary relation $P$ with which $\succsim$ is $P$-intrinsic consistent $(P$-instrumental consistent in the case of Theorem 4.2) by defining it as $P=\{(x, y)$ such that $\{x, y\} \succ\{y\}\}$, which by RI implies $\{x, y\} \sim\{x\}$. Therefore, SC implies that the absence of this binary relation $P$ is aversive $(\{x\} \succ\{x, y\}$ and $\{y\} \succ\{x, y\})$.

Definitions 4.1 and 4.2 diverge, however, on how the addition of an alternative affects a set which is not a singleton. This is reflected in the corresponding axioms. As the proofs show, both characterizations are the result of the combined effect of all the axioms, but in the next paragraphs we try to provide an intuition about how the different axioms enter the results.

When modeling how an alternative can improve a set, Theorem 4.1 assumes axiom MC, while Theorem 4.2 assumes MR. MC states that an alternative has to improve all the 
singletons to improve a set and it is logically stronger than MR. MC is linked with the definition of intrinsic consistency, which says that an alternative has to dominate all the alternatives of a set in order to improve it. MR is linked with instrumental consistency because Definition 4.2 states that the improvement provided by $x$ must not be absorbed by $y$, and this happens when $y$ dominates $x$, which is explicitly excluded by axiom MR.

The two theorems also differ as to the axioms used to model a worsening. Theorem 4.1 uses CE, which is consistent with the idea in Definition 4.1 that it is sufficient that $x$ is incomparable with one alternative to worsen the set. However, CC and CR (used in Theorem 4.2) are more demanding with respect to the conditions on $x$ to worsen a set $A$, in concordance with the conditions that Definition 4.2 imposes to worsen a set (no alternative in $A$ dominates $x$ and $x$ does not dominate any alternative in the $P$-maximal set of $A$ ).

Finally, there are two different axioms that refer to situations where the addition of an alternative does not affect a set: IC and IA. IC (used in Theorem 4.2) states that if an alternative $x$ does not affect a set and dominates some other alternative $y$, then neither $y$ should affect the set. Notice that, in the particular case where $x$ belongs to the set, this axiom means that $y$ does not affect the set whenever there is some dominating alternative inside. IC is logically weaker than its counterpart axiom in Theorem 4.1, IA, which considers more situations in which the addition of an alternative should not affect a set. This is in concordance with Definitions 4.1 and 4.2. The conditions in Definition 4.1 for a new alternative to be inocuous (i.e. being comparable with every alternative in the set but dominated by some existing alternative) are more demanding than in Definition 4.2 (i.e. the existence of some dominating alternative in the set).

Summing up, Definition 4.1 imposes more demanding conditions than Definition 4.2 to determine that an alternative improves or does not affect a set. This is reflected by the assumption of stronger axioms modeling these situations. However Definition 4.1 imposes weaker conditions to determine that an alternative worsens a set, which is again reflected in the characterization by the weaker axioms used to model this.

Another remarkable difference between the two characterizations is that, if $\succsim$ satisfies the axioms in Theorem 4.2, then the underlying binary relation $P$ has to be transitive while this is not the case in Theorem 4.1, where $P$ can even be cyclic. ${ }^{8}$ This fact is a reflection of our interpretation about the cognitive abilities assumed by each definition (see point

\footnotetext{
${ }^{8}$ Notice that it is the absence of $P$ and not $P$-cycles that is linked with the aversion to incomparability: If $X=\{x, y, z\}, x P y, y P z$ and $z P x$ we have that $A \cup\{x\} \succsim A$ for all $A \in \pi(X)$ and for all $x \in X \backslash A$, that is, there is no aversion to incomparability.
} 
(iv) above). Being able to identify the $P$-maximal subset of every set in the instrumental approach indirectly leads to the need of $P$ to be transitive. For the statements in Definition 1 to be possible, it is not necessary (but it is not precluded) that $P$ be transitive. There the agent is just supposed to perform pairwise comparisons between the additional alternative and the existing ones, which is a less elaborated task than identifying previously the $P$ maximal set. Thus, even agents whose preference over the alternatives is intransitive can behave consistently with Definition 1, while the statements in Definition 2 are only possible if $P$ is transitive. In the next subsection we provide a result where the assumptions in Theorem 4.1 are plausibly strengthened in such a way that the resulting $P$ turns out to be transitive.

\subsection{Transitive rules}

Definitions 1 and 2 only establish comparisons between pairs of sets $(A, B)$ where $B=$ $A \cup\{x\}$ for some $x \notin A$. As previously pointed out, such a reduced approach aims to isolate, as accurately as possible, the content and meaning of the idea of aversion to incompleteness from other "noisy" factors that arise when more dissimilar sets are compared. As a consequence, wide classes of rules are axiomatically characterized as being $P$-consistent, but at the cost of a reduced insight about how those rules should look like in general.

In this subsection we impose more structure on $\succsim$ in order to provide more insightful criteria to compare sets. In particular, we analyze the consequences of assuming transitivity of $\succsim$ and a minimal requirement of consistency, called Extension, which is widespread in the ranking sets literature (see, for example, the survey by Barberà et al. (2004)). This condition allows to compare singletons in such a way that the relative ranking between singletons should coincide with their ranking in terms of $P$.

- Extension (E): $\forall x, y \in X, x P y$ if and only if $\{x\} \succ\{y\}$

Assuming transitivity of $\succsim$, even though it is not necessary for inferring aversion to incompleteness, leads to the fact that the binary relation, $P$, with which $\succsim$ is $P$-intrinsic consistent, is acyclic:

Corollary 1. Let $\succsim$ be a binary relation defined on $\pi(X)$. Let $P$ be a binary relation defined on $X$ such that $\succsim$ is $P$-intrinsic consistent. If $\succsim$ is transitive then $P$ is acyclic. 
Proof: Assume that $\succsim$ is $P$-intrinsic consistent and transitive but $P$ is not acyclic. This implies that there exist $x_{1}, x_{2}, \ldots x_{n}$ such that $x_{1} P x_{2} P \ldots x_{n} P x_{1}$. Given that $\succsim$ is $P$ intrinsic consistent, we have by definition: $\left\{x_{1}\right\} \succsim\left\{x_{1}, x_{2}\right\} \succsim \ldots \succsim\left\{x_{1}, x_{2}, \ldots, x_{n}\right\}$ and $\left\{x_{1}, x_{2}, \ldots, x_{n}\right\} \succsim\left\{x_{1}, \ldots, x_{n-2}, x_{n}\right\} \succsim \ldots \succsim\left\{x_{1}, x_{n}\right\}$. Then, by transitivity of $\succsim,\left\{x_{1}\right\} \succsim$ $\left\{x_{1}, x_{n}\right\}$. Also by being $\succsim P$-intrinsic consistent we have $\left\{x_{1}, x_{n}\right\} \succ\left\{x_{1}\right\}$, reaching a contradiction.

If, in addition to transitivity of $\succsim, E$ is also assumed, then $P$ turns out to be transitive:

Corollary 2. Let $\succsim$ be a binary relation defined on $\pi(X)$. Let $P$ be a binary relation defined on $X$ such that $\succsim$ is P-intrinsic consistent. If $\succsim$ is transitive and satisfies $E$ then $P$ is transitive.

Proof: By theorem 4.1 we know that if $\succsim$ is $P$-intrinsic consistent then it satisfies RI. Assume $x P y$ and $y P z$. Then, by the definition of $P$ and RI, we have $\{x\} \sim\{x, y\} \succ\{y\} \sim$ $\{y, z\} \succ\{z\}$. By transitivity we have $\{x\} \succ\{z\}$, which by E implies $x P z$.

Notice that, as proved in Theorem 4.2, when $\succsim$ is $P$-instrumental consistent, it is not necessary to assume its transitivity for the arising $P$ to be also transitive.

Assuming that $\succsim$ is transitive also provides more insights about its general structure. This is shown in the next corollaries, for which an additional piece of notation is needed. For all $A \in \pi(X)$ let $\otimes(A)=\#\{(x, y): x \neq y, x, y \in A$ and $x \otimes y\}$ (that is, $\otimes(A)$ counts the number of pairwise incomparabilities in $A)$.

Corollary 3. Let $\succsim$ be a transitive, $P$-intrinsic consistent binary relation defined on $\pi(X)$. Then, $\forall A, B \in \pi(X)$ such that $A \subset B$ the following conditions hold:

(i) $[\otimes(A)=\otimes(B)$ and $\exists b \in B \backslash A$ such that $b P a$ for all $a \in A]$ implies $B \succ A$

(ii) $[\forall b \in B \backslash A$ there exists $a \in A$ such that $a \otimes b)]$ implies $A \succ B$

(iii) $\left[\max _{P}(A)=\max _{P}(B)\right.$ and $\left.\otimes(A)=\otimes(B)\right]$ implies $A \sim B$.

Moreover, $P$ is acyclic and if $\succsim$ satisfies $E$ then $P$ is transitive.

Corollary 4. Let $\succsim$ be a transitive, P-instrumental consistent binary relation defined on $\pi(X)$. Then, $\forall A, B \in \pi(X)$ such that $A \subset B$ the following conditions hold:

(i) $\left[\# \max _{P}(B \backslash A)=1\right.$ and $\max _{P}(B \backslash A) P a$ for some $\left.a \in \max _{P}(A)\right]$ implies $B \succ A$

(ii) $\left[a \otimes b \forall b \in \max _{P}(B \backslash A) \forall a \in \max _{P}(A)\right]$ implies $A \succ B$

(iii) $\left[\max _{P}(A)=\max _{P}(B)\right]$ implies $A \sim B$.

Moreover $P$ is transitive. 
The proofs of Corollaries 3 and 4 are presented in the Appendix. In words, Corollary 3 states that any transitive $P$-instrinsic rule should satisfy that, when adding new alternatives to a set (i) if at least one of them dominates all the existing ones and none of them involves additional incomparabilities, then the enlarged set is better; (ii) if all the additional ones are incomparable with at least one existing alternative, then the enlarged set is worse; and (iii) if the new alternatives do not contribute to the maximal set and do not incorporate additional incomparabilities, then the enlarged set is indifferent. Analogously, Corollary 4 states that any transitive $P$-instrumental rule should satisfy that, when adding new alternatives to a set (i) if the set of new alternatives are not pairwise incomparable and at least one is better than an existing maximal one, then the enlarged set is better; (ii) if the maximal set of the new alternatives are all incomparable with all the alternatives in the existing maximal set, then the enlarged set is worse; and (iii) if all the new alternatives are dominated by some existing one, then the enlarged set is indifferent.

Next we show, for illustrative purposes, some examples of $P$-consistent rules that are in the domain of Corollaries 3 and 4 respectively.

Let $\succcurlyeq_{1}$ be defined by:

$$
\forall A, B \in \pi(X)\left\{\begin{array}{c}
A \succ B \text { if } \otimes(A)<\otimes(B) \text { or } \\
\otimes(A)=\otimes(B) \text { and } \exists a \in \max _{P}(A) \text {, s.t } a P b \forall b \in \max _{P}(B) \\
A \sim B \text { if } \max _{P}(A)=\max _{P}(B) \text { and } \otimes(A)=\otimes(B)
\end{array}\right.
$$

Let $\succcurlyeq_{2}$ be defined by:

$\forall A, B \in \pi(X)\left\{\begin{array}{c}A \succ B \text { if } \otimes \max _{P}(A)<\otimes \max _{P}(B) \text { or } \\ \otimes\left(\max _{P}(A)\right)=\otimes\left(\max _{P}(B)\right) \text { and } \exists a \in \max _{P}(A) \text {, s.t } a P b \text { for some } b \in \max _{P}(B) \text { and } \\ \nexists b \in B \text { s.t } b P a \text { for some } a \in \max _{P}(A) \\ A \sim B \text { if } \max _{P}(A)=\max _{P}(B)\end{array}\right.$

$\succcurlyeq_{1}$ and $\succcurlyeq_{2}$ are both lexicographic rules. They first minimize the number of incomparabilities either in the $P$-maximal set $\left(\succcurlyeq_{2}\right)$ or in the whole set $\left(\succcurlyeq_{1}\right)$. If the number of incomparabilities is equal, then they look at the alternatives contained in the $P$-maximal 
subset. According to $\succsim_{1}$ a set $A$ is preferred to another $B$ if $\max _{P}(A)$ contains an alternative that is preferred to all the alternatives in $\max _{P}(B)$. According to $\succsim_{2}$ there must be an alternative in $\max _{P}(A)$ that is preferred to some other alternative in $\max _{P}(B)$ and the reverse is not true. If both $P$-maximal subsets are equal, the two sets are indifferent under the two rules. Otherwise they are not comparable.

The next examples, besides being transitive rules, are also complete:

Let the anti-cardinalist ranking $\succcurlyeq_{\#} \subseteq \pi(X) \times \pi(X)$ be defined by: $\forall A, B \in \pi(X)$, $A \succcurlyeq_{\#} B$ if and only if $\# A \leq \# B$.

Let $R$ be a complete preorder defined on $X$ and let the indirect utility criterion $\succcurlyeq_{I U \subseteq} \subseteq$ $\pi(X) \times \pi(X)$ be defined by: $\forall A, B \in \pi(X), A \succcurlyeq_{I U} B$ if and only if, for all $a \in \max _{R}(A)$, $b \in \max _{R}(B), a R b$.

The anticardinalist ranking considers that a set is better than another when it contains less alternatives and two sets are indifferent when they have the same number of alternatives. $\succcurlyeq_{I U}$ is the classical criterion of the consumer theory, which evaluates sets exclusively by means of their best alternatives.

It can be easily checked that $\succcurlyeq_{\#}$ is both $P$-intrinsic and $P$-instrumental consitent under $P=\emptyset$ and that $\succcurlyeq_{I U}$ is both, $P$-intrinsic and $P$-instrumental consitent when $P$ is a linear order.

\section{Aversion to incompleteness and compatibility with prefer- ence for flexibility}

The preference for flexibility and the economic value of freedom of choice are important concepts that have also been discussed by means of the axiomatic analysis of rankings over opportunity sets. The ideas of intrinsic and instrumental value of freedom of choice have already been roughly explained in Section 4.1. At an elementary formal level, the difference between them is that, according to the intrinsic value of freedom of choice, even if $\neg(x P y)$, $\{x, y\} \succ\{x\}$ and $\{x, y\} \succ\{y\}$, while according to the instrumental value of freedom of choice, $\neg(x P y)$ implies $\neg(\{x, y\} \succ\{x\})$.

Preference for flexibility arises in two-stage decision processes where the individual has, at the first stage, uncertainty about her future preferences and therefore displays a preference for preserving more options (choosing larger opportunity sets) as a means to be able 
to choose a better final alternative in the second stage when uncertainty is expected to be dissipated (see Kreps (1979), Nehring (1999) or Arlegi and Nieto (2001a)).

From a conceptual point of view, preference for flexibility can be understood as a particular form of the instrumental value of freedom of choice in an uncertainty scenario: preserving an option $x$ in the opportunity set at stage 1 is only valuable as long as there is some future state of nature where $x$ is the best alternative. A formal consequence of preference for flexibility is that, if $P$ is interpreted as an indubitable preference, even if $\neg(x P y)$, it could be the case that $\{x, y\} \succ\{y\}$, as it was the case in the intrinsic approach to the value of freedom of choice. ${ }^{9}$

In this section we discuss the relationship of our approach with the freedom of choice and preference for flexibility ideas.

Our two definitions (instrumental and intrinsic) of aversion to incompleteness absolutely disregard the hypothesis of an intrinsic value of freedom of choice. In our model, the mere availability of more alternatives has never an intrinsic value. In Definitions 1 and 2, having more options can only have an instrumental value: Having more alternatives when a better option has been added to a set involves an improvement only because the new alternative allows for the set to provide a higher indirect utility. In terms of the elementary comparisons noted above, under Definitions 1 and $2, \neg(x P y)$ implies $\neg(\{x, y\} \succ\{y\})$, in opposition to the models of intrinsic value of freedom of choice.

As to the compatibility of our approach with the preference for flexibility assumption, from a formal point of view, aversion to incompleteness and preference for flexibility produce opposite results: if $\neg(x P y)$ and $\neg(y P x)$, under the preference for flexibility approach, $\{x, y\} \succ\{x\}$ and $\{x, y\} \succ\{y\}$, while in our aversion to incompleteness approach what happens is just the opposite, that is, $\{x\} \succ\{x, y\}$ and $\{y\} \succ\{x, y\}$.

Does this mean that aversion to incompleteness and preference for flexibility are incompatible phenomena? Our view is that they are compatible. The key idea is that the absence of a preference has a different meaning in the preference for flexibility approach and in the aversion to incompleteness one. We show that it is possible to subsume in a unique model both ideas by an appropriate formalization of the absence of preference by means of two distinct binary relations of incomparability, following the distinction between tentative incompleteness and assertive incompleteness posed by (Sen, 1997, pp. 763-764) :

\footnotetext{
${ }^{9}$ In fact, Arlegi and Nieto $(2001 \mathrm{a}, \mathrm{b})$ show that rankings of opportunity sets that are proposed in the literature as displaying the intrinsic value of freedom of choice can plausibly be re-interpreted as the result of preference for flexibility.
} 
"It is useful to consider the distinction between: tentative incompleteness, when some pairs of alternatives are not yet ranked (though they may all get ranked with more deliberation or information), and assertive incompleteness, when some pair of alternatives is asserted to be "non-rankable." Assertive incompleteness is the claim that the failure of completeness is not provisional - waiting to be resolved with, say, more information, or more penetrating examination. The partial ranking, or the inexhaustive partitioning, may simply not be "completable", and affirming that some $x$ may not be rankable vis-a-vis some y may be the right answer in these cases."

We formally describe Sen's difference between tentative and assertive incompleteness by means of two different binary relations, $\otimes_{1}, \otimes_{2} \in X^{2}$ such that, $\forall x, y \in X, x \otimes_{1} y$ is interpreted as an incomparability between $x$ and $y$ with the expectation that at a later stage incomparability will be solved (tentative incompleteness), and $x \otimes_{2} y$ is interpreted as an incomparability between $x$ and $y$ without such an expectation (assertive incompleteness). The binary relation $P \in X^{2}$ maintains the interpretation of an indubitable preference used throughout the previous sections.

Given the meaning attached to $P, \otimes_{1}$ and $\otimes_{2}$, we assume that $P$ is asymmetric, $\otimes_{1}$ and $\otimes_{2}$ are symmetric, $\otimes_{1} \cap \otimes_{2}=\emptyset$ and $P=X^{2} \backslash\left\{\otimes_{1} \cup \otimes_{2}\right\}$. When $P, \otimes_{1}$ and $\otimes_{2}$ satisfy such conditions we say that " $\left(P, \otimes_{1}, \otimes_{2}\right)$ is a preference with a structured incompleteness". By this we mean that the absence of preference has the particular structure given by the properties that we have assumed on $P, \otimes_{1}$ and $\otimes_{2}$. In some cases we will assume that $P$ is, in addition, transitive. In that case we will say that " $\left(P, \otimes_{1}, \otimes_{2}\right)$ is a transitive preference with a structured incompleteness".

What we explore next is, like in the preference for flexibility models, how the preference for sets should be at stage 1 considering that: the individual (i) has a preference to preserve alternatives that might be optimal at stage 2 , when the final single choice has to be made (preference for flexibility) and (ii) is able to anticipate the aversion to incomparability that she would face at stage 2 if alternatives are assertively incomplete.

According to the interpretation of $P, \otimes_{1}$ and $\otimes_{2}$, the following are the different possibilities regarding elementary comparisons of $\{x, y\}$ with $\{x\}$ and with $\{y\}$ :

If $x P y$ then $\{x, y\} \sim\{x\},\{x, y\} \succ\{y\}$ and $\{x\} \succ\{y\}: y$ does not add any value to $x$ but $x$ adds value to $y$.

If $x \otimes_{1} y$ then $\{x, y\} \succ\{x\}$ and $\{x, y\} \succ\{y\}$ : the decision maker does not expect to experience a psychological cost when the final choice has to be made and the availability of both options provides flexibility. 
If $x \otimes_{2} y$ then $\{x\} \succ\{x, y\}$ and $\{y\} \succ\{x, y\}$ : the decision maker anticipates the aversion to have both alternatives. Flexibility has not any value in this case. Flexibility is valuable as long as it is a means to choose a better option at the second stage of the decision process once uncertainty is dissipated, but in this case there is no expectation of having a best option at that stage.

When more general comparisons of the type $A \cup\{x\}$ versus $A$ are in order (like in the previous sections), then the addition of $x$ to $A$ may involve conflicting effects, for example, if there are alternatives in $A$ with which $x$ is related by means of $\otimes_{1}$ but also other alternatives with which $x$ is related by means of $\otimes_{2}$.

Basically, the distinction between the two types of incomparabilities is what allows us to depart from the impossibility result in Pejsachowicz and Toussaert (2017), where indecisiveness in the preferences is shown to be incompatible with the preference for smaller menus. ${ }^{10}$ Leaving aside some nonessential formal differences between the two approaches, a key reason for the impossibility to hold is the assumption that incomparability will eventually dissipate if more options are preserved. What we show is that when broader scenarios for incompleteness are considered, then both preference for flexibility and preference for smaller menus, are compatible.

Thus, the aim of the following results is to show formally this compatibility, either under the intrinsic or instrumental interpretation of the aversion to incompleteness. We provide axiomatic characterizations on the basis of the axioms presented in Section 3. Given that the absence of $P$ may hold in two ways (either by means of $\otimes_{1}$ or by means of $\otimes_{2}$ ), the notion of $P$-consistency in this, more complex, framework needs to be re-stated as $\left(P, \otimes_{1}, \otimes_{2}\right)$ consistency.

Definition 3. Let $\left(P, \otimes_{1}, \otimes_{2}\right)$ be a preference with a structured incompleteness. A reflexive binary relation $\succsim \subseteq \pi(X) \times \pi(X)$ is $\left(P, \otimes_{1}, \otimes_{2}\right)$-consistent with the intrinsic aversion to incomplete preferences and the preference for flexibility, henceforth $\left(P, \otimes_{1}, \otimes_{2}\right)$-intrinsic consistent, if it satisfies the following condition:

\footnotetext{
${ }^{10}$ Indecisiveness in Pejsachowicz and Toussaert (2017) is understood as incompleteness of the preferences over sets, but this can be naturally translated to our context in the particular case where those sets are singletons.
} 
$\forall A \in \pi(X), \forall x \in X \backslash A\left\{\begin{aligned} & A \cup\{x\} \succ A \text { if for all } a \in A \text {, either } a \otimes_{1} x \text { or } x P a \\ & A \succ A \cup\{x\} \text { if } \exists a \in A \text { such that } a \otimes_{2} x \\ & A \cup\{x\} \sim A \text { if } \nexists a \in A \text { such that } a \otimes_{2} x \text { and } \exists a \in A \text { such that aPx }\end{aligned}\right.$

Definition 4. Let $\left(P, \otimes_{1}, \otimes_{2}\right)$ a transitive preference with a structured incompleteness. A reflexive binary relation $\succsim \subseteq \pi(X) \times \pi(X)$ is $\left(P, \otimes_{1}, \otimes_{2}\right)$-consistent with the instrumental aversion to incomplete preferences and the preference for flexibility, henceforth, $\left(P, \otimes_{1}, \otimes_{2}\right)$ instrumental consistent, if it satisfies the following condition:

$\forall A \in \pi(X), \forall x \in X \backslash A\left\{\begin{array}{c}A \cup\{x\} \succ A \text { if }\left[\exists a \in \max _{P}(A) \text { s.t } x P a \text { or } x \otimes_{1} a\right] ;\left[\nexists a \in \max _{p}(A) \text { s.t } a P x\right] \\ \text { and }\left[\forall a^{*} \in \max _{P}(A) \text { s.t } a^{*} \otimes_{2} x \nexists a \in \max _{P}(A) \text { s.t } a \otimes_{1} a^{*}\right] \\ A \succ A \cup\{x\} \text { if } x \otimes_{2} \text { a for all } a \in \max _{P}(A) \\ A \cup\{x\} \sim A \text { if } \exists a \in A \text { such that aPx }\end{array}\right.$

It is easy to check that, when $\otimes_{1}$ is empty, Definition 3 coincides with Definition 1 $(P$-intrinsic consistency) and Definition 4 coincides with Definition 2 ( $P$-instrumental consistency). Also, when $\otimes_{2}$ is empty, both Definitions 3 and 4 , coincide with the definition of $P$-consistency with the preference for flexibility in Arlegi and Nieto (2001a). ${ }^{11}$

As already pointed, any rule that tries to make compatible the preference for flexibility and the aversion to incompleteness ideas should necessarily solve, in some way, a trade-off between them. Obviously this issue might be solved in different ways, but given that there could be cross effects between $\otimes_{1}$ and $\otimes_{2}$, it is not immediate that every solution is able to solve the trade-offs in a consistent way. The two rules above do it as follows:

The rule defined in Definition 3 treats asymmetrically the two phenomena. For a new alternative, $x$, to improve a given set it is necessary that, for every alternative $a$ in $A$, either $x$ dominates $a$ or provides flexibility to $a$. But for $x$ to worsen $A$ it is sufficient that there

\footnotetext{
${ }^{11}$ However, form a formal point of view, preference for flexibility in our model involves less structure than in Kreps (1979), since it just applies to particular comparisons of pairs $(A, B)$ such that $B=A \cup\{x\}$ for some $x \notin A$.
} 
exists at least at least one alternative in $A$ with which it is assertively incomparable (it involves a pairwise aversion to incomparability).

The rule defined in Definition 4 is different in several aspects. First, it focusses on the effect of the addition of $x$ on the set of $P$-maximal alternatives. Also, in order to declare that $x$ worsens $A$ it is necessary that it is assertively incomparable with all the alternatives in $\max _{P}(A)$. Finally, in order to declare that $x$ improves $A$, three conditions should hold: first, either $x$ is preferred to some alternative in $\max _{P}(A)$, or adds flexibility to some alternative in $\max _{P}(A)$; secondly, no other alternative in $A$ should be better than $x$, and thirdly, if $x$ involves some incomparability aversion with respect to some alternative in $a^{*} \in \max _{P}(A)$, $a^{*}$ should not add pairwise flexibility to any other alternative in $a^{*} \in \max _{P}(A)$, that is, the addition of $x$ should not "cancel" the flexibility that $a^{*}$ provides with respect to some other alternative in $\max _{P}(A)$.

For the axiomatic characterization of the two rules of Definitions 3 and 4 we will use the following weaker version of axiom RI:

- Weak Revealed Indifference (WRI)

$$
\forall x, y \in X,\{x, y\} \sim\{y\} \Rightarrow\{x, y\} \succ\{x\} \text { and }\{x, y\} \succ\{y\} \Rightarrow\{x, y\} \succsim\{x\}
$$

Theorem 5.1. Let $\succsim$ be a binary relation defined on $\pi(X)$. There exists a preference with a structured incompleteness $\left(P, \otimes_{1}, \otimes_{2}\right)$ such that $\succsim$ is $\left(P, \otimes_{1}, \otimes_{2}\right)$-intrinsic consistent if and only if $\succsim$ satisfies WRI, SC, IA, MC and CE.

Theorem 5.2. Let $\succsim$ be a reflexive binary relation defined on $\pi(X)$. If $\succsim$ satisfies $W R I$, $S C, C C, I C, M R, M C$ and $C R$ then there exists a transitive preference with a structured incompleteness $\left(P, \otimes_{1}, \otimes_{2}\right)$ such that $\succsim$ is $\left(P, \otimes_{1}, \otimes_{2}\right)$-instrumental consistent.

Theorem 5.3. Let $\succsim$ be a reflexive binary relation defined on $\pi(X)$. If there exists a transitive preference with a structured incompleteness $\left(P, \otimes_{1}, \otimes_{2}\right)$ such that $\succsim$ is $\left(P, \otimes_{1}, \otimes_{2}\right)$ instrumental consistent then $\succsim$ satisfies WRI, SC, CC, IC, and MC.

Proposition 3. Axioms WRI, SC, IA, MC and CE are independent.

Proposition 4. Axioms WRI, SC, CC, IC, MR, MC and CR are independent.

Like Definitions 1 and 2, Definition 3 is exhaustive in the sense that, for all $A \in \pi(X)$, for all $x \notin A$, it determines how to rank $A$ and $A \cup\{x\}$. However, according to Definition 4 there are situations where there are cross effects, so that the rule does not compare $A$ and 
$A \cup\{x\}$. In particular, this is the case exactly when: (i) there does not exist $z \in A$ such that $z P x$ and (ii) there exist $a, a^{*} \in \max _{P}(A)$ such that either $x P a$ or $x \otimes_{1} a ; x \otimes_{2} a^{*}$; and $a \otimes_{1} a^{*}$. This is the reason for not obtaining a characterization theorem for Definition 4 : the ambiguity in some situations does not allow to guarantee that MR and CR are fulfilled.

When comparing the axiomatic characterization of Theorem 4.1 ( $P$-intrinsic consistency) with that of Theorem 5.1 (incorporating the preference for flexibility idea), the only difference is the weakening of RI into WRI. Similarly, when comparing Theorem 4.2 and 5.2 we find that RI is weakened into WRI both as a necessary and a sufficient axiom (Theorems 5.2 and 5.3). That is, in both cases, the (apparently) slight weakening of RI into WRI is what gives rise to the possibility of flexibility to be valuable. Particularly, RI was inferring $x P y$ from either $\{x, y\} \succ\{y\},\{x, y\} \sim\{x\}$ or both. However, WRI only infers $x P y$ if $\{x, y\} \sim\{x\}$. The reason is that, under the preference for flexibility assumption, the absence of preference may lead to $\{x, y\} \succ\{x\}$.

This gives an idea of the relevance of RI-like axioms. They look simple and are elementary but encapsulate the admissibility of different interpretations for the absence of preference. RI leads to the unique interpretation of the absence of preference as costly incomparability. WRI gives rise to the interpretation of the absence of preference as "valuable" incomparability (in terms of flexibility). There is a third interpretation for the absence of preference that has not appeared in the precedent sections: that of an indubitable (or absolutely certain) indifference. In fact it is possible to design an alternative weakening of $\mathrm{RI}$ that gives rise to such interpretation. Let us consider the following axiom:

- Weak Revealed Indifference 2 (WRI2)

$$
\forall x, y \in X,\{x, y\} \sim\{y\} \Rightarrow\{x, y\} \succsim\{x\} \text { and }\{x, y\} \succ\{y\} \Rightarrow\{x, y\} \sim\{x\}
$$

Note that WRI2 is satisfied by the standard indirect utility criterion. In particular, when $\{x, y\} \sim\{y\}$ WRI2 admits that $\{x, y\} \sim\{x\}$, which would correspond to the situation where both $x$ and $y$ are indifferent with absolute certainty. ${ }^{12}$

Given that RI is the intersection of WRI and WRI2, for a model to comprehend the three interpretations of the absence of preference we would need an axiom consisting of the union of WRI and WRI2, that is:

\footnotetext{
${ }^{12}$ The details go beyond the reasonable length of this work but, in principle, it would be possible to obtain characterization results analogous to Theorems 5.1 and 5.2 making compatible costly incomparabilities with the possibility of absolutely certain indifferences.
} 
- $\forall x, y \in X,\{x, y\} \succsim\{y\} \Rightarrow\{x, y\} \succsim\{x\}$

In sum, the part of RI stating that $\{x, y\} \succ\{y\}$ implies $\{x, y\} \sim\{x\}$ rules out the possibility of interpreting the absence of preference as a "valuable" incomparability. The inverse part of RI stating that $\{x, y\} \sim\{x\}$ implies $\{x, y\} \succ\{y\}$ rules out the possibility of interpreting the absence of preference as an absolutely certain indifference. A question naturally arises: What would ruling out the possibility of interpreting the absence of preference as a costly incomparability amount to? The answer is not in any RI-like axiom. Models of preference of flexibility, or freedom of choice, as well as the indirect utility criterion respects a basic monotonicity condition which is not satisfied by our model of incompleteness:

- $\{x, y\} \succsim\{x\} \forall x, y \in X^{13}$

This condition, in the case of the models of the intrinsic value of freedom of choice takes the stronger form $\{x, y\} \succ\{x\} \forall x, y \in X$ (Pattanaik and Xu (1990) "simple monotonicity's" axiom). ${ }^{14}$

\section{Conclusions}

We developed an analysis of aversion to incomplete preferences by means of the axiomatic characterization of two different rules that encapsulate two different ways of conceiving incompleteness (Theorems 4.1 and 4.2). The very general idea is that the addition of a better alternative to a set improves it, the addition of a worse alternative does not affect the set, and the addition of an alternative that involves some incomparability worsens the set. However we distinguish two types of consistency: $P$-intrinsic consistency arises when incomparability affects any alternative of the set and $P$-instrumental consistent arises when incomparability affects alternatives in the $P$-maximal subset.

The two proposed rules determine comparisons among a reduced class of pairs of sets and allow for any extension of them to be intransitive and/or incomplete. The additional

\footnotetext{
${ }^{13}$ In Kreps (1979) model of preference for flexibility this condition takes the more general form $A \subseteq B$ implies $B \succsim A$.

${ }^{14}$ Eliaz and Ok (2006) also distinguish between an indifference and an indecisiveness binary relation, proposing a rationalizability of both by means of a weaker version of the weak axiom of revealed preference. Our approach is related to theirs as long as we distinguish different ways in which the absence of preference may hold and claim the relevance of the distinction, but we do not obtain it as an induction from a choice function but as an elicitation from preferences over elementary sets of the type $\{x, y\}$ versus $\{x\}$.
} 
assumption of transitivity on the binary relation over the sets allows to gain some insight about the general structure of the rule and guarantees that the binary relation $P$ with which it is consistent is also transitive. Moreover we show that that complete and transitive rankings of opportunity sets can also be $P$-consistent in any of the two senses.

Finally, we discuss the compatibility of the aversion to incompleteness and the preference for flexibility phenomena. In principle they act in opposite directions, but we show that they are compatible if we adequately distinguish between different types of incomparability, recalling Sen (1997) distinction between assertive and tentative incompleteness. We propose a model that integrates both types of incomparability and we characterize axiomatically two rules that are $P$-consistent with both the aversion to incompleteness and compatible with the preference for flexibility idea (Theorems 5.1 and 5.2). The axiomatic characterizations reveal that axiom RI, though being rather elementary, is playing an important role in relation with the admissibility of different interpretations of the absence of preference. In particular, we show how alternative weakenings of RI may give rise to the admissibility of the interpretation of the absence of preference as valuable incomparability (in terms of preference for flexibility) and/or as a standard indifference relation.

\section{$7 \quad$ Appendix}

\subsection{Proof of Theorem 4.1}

In order to prove the sufficient part of the Theorem we have to prove that if $\succsim$ is $P$-intrinsic consistent with some asymmetric binary relation $P$ then $\succsim$ satisfies RI, SC, MC, IA and CE.

- RI: $\{x, y\} \succ\{y\}$ implies, by the definition of $P$-intrinsic consistency, that $x P y$, which again by the definition of $P$-intrinsic consistency implies $\{x, y\} \sim\{y\}$, and vice versa.

- SC: By the definitions of $P$ and $\otimes$, given any pair $x, y \in X$ either $x P y, y P x$ or $x \otimes y$. By Definition 1, if $x P y$ then $\{x, y\} \sim\{x\}$; if $y P x$ then $\{x, y\} \succ\{x\}$; and if $x \otimes y$ then $\{x\} \succ\{x, y\}$.

- MC: Let $A \in \pi(X)$ and let $x \in X \backslash A$. Assume that $\{x, a\} \succ\{a\}$ for all $a \in A$. This implies, by Definition 1 , that $x P a$ for all $a \in A$, which by the same definition implies $A \cup\{x\} \succ A$. 
- IA: Let $A \in \pi(X)$ and let $x \in X \backslash A$. Assume that $\{a, x\} \succsim\{a\} \forall a \in A$. Thus, $\nexists a \in A$ such that $\{a\} \succ\{a, x\}$, which by Definition 1 implies (i): $\nexists a \in A$ s.t $a \otimes x$. Assume that, in addition, $\exists a^{*} \in A$ such that $\left\{a^{*}, x\right\} \sim\left\{a^{*}\right\}$. This, by Definition 1, implies (ii): $a^{*} P x$. Given that (i) and (ii) hold, by Definition 1, $A \cup\{x\} \sim A$.

- CE: Assume that $A \succ A \cup\{x\}$. By Definition 1 this implies that there exists $a \in A$ such that $x \otimes a$. Therefore, if $A \subseteq B$ then there exists $b \in B,(b=a)$ such that $x \otimes b$, which by Definition 1 implies $B \succ B \cup\{x\}$.

In order to prove the necessary part of the theorem, assume that a binary relation $\succsim$ satisfies all the axioms of Theorem 4.1. Then there exists an asymmetric binary relation $P$ defined on $X$ with which $\succsim$ is $P$-intrinsic consistent.

We will show that, in particular, $\succsim$ is consistent with the relation $P$ defined by: $P=$ $\{(x, y)$ such that $\{x, y\} \succ\{y\}\}$.

First, we have to prove that $P$ is asymmetric: Assume $x P y$, then by the definition of $P,\{x, y\} \succ\{y\}$. Assume that $P$ is not asymmetric and therefore $y P x$. Then $\{x, y\} \succ\{x\}$, but this is in contradiction with RI.

Now, take $A \subseteq \pi(X)$ and $x \in X \backslash A$ :

(i) Assume that $x P a$ for all $a \in A$. This, by the definition of $P$, implies $\{a, x\} \succ\{a\}$ $\forall a \in A$, which by MC implies $A \cup\{x\} \succ A$.

(ii) Assume, now, that there exists $a$ in $A$ such that $x \otimes a$. Then, by the definition of $\otimes$, we know that $\neg(a P x)$ and $\neg(x P a)$. Given that $\neg(a P x)$, by the definition of $P, \neg(\{a x\} \succ\{x\})$, which by RI implies $\neg(\{a x\} \sim\{a\})$. On the other hand, given that $\neg(x P a)$, again by the definition of $P, \neg(\{a x\} \succ\{a\})$. By SC, given that $\neg(\{a x\} \sim\{a\})$ and $\neg(\{a x\} \succ\{a\})$, we have that $\{a\} \succ\{a x\}$. Since $\{a\} \subseteq A$, by CE, $A \succ A \cup\{x\}$.

(iii) Assume that none of the two assumptions in (i) and (ii) hold. Therefore, for all $a \in A$, either $a P x$ or $x P a$ and $\exists a^{*} \in A$ such that $a^{*} P x$. By the definition of $P$ we have that $\left\{a^{*}, x\right\} \succ\{x\}$ and by $\mathrm{RI}\left\{a^{*}, x\right\} \sim\left\{a^{*}\right\}$. Also, for all $a \in A$ either $a P x$, which as we have shown implies $\{a, x\} \sim\{a\}$, or $x P a$ which by the definition of $P$ implies $\{a, x\} \succ\{a\}$. Therefore, $\forall a \in A\{a, x\} \succsim\{a\}$. Given that $\left\{a^{*}, x\right\} \sim\left\{a^{*}\right\}$ and $\forall a \in A\{a, x\} \succsim\{a\}$, by IA we have $A \cup\{x\} \sim A$.

To prove that the binary relation $P$ with which $\succsim$ is $P$-consistent is unique, assume that there exists another binary relation $P^{\prime} \neq P$ with which $\succsim$ is $P^{\prime}$-consistent. $P \neq P^{\prime}$ implies that there exist $x, y \in X$ such that $x P^{\prime} y$ and $\neg(\{x, y\} \succ\{y\})$. In this case, by the definition of $P^{\prime}$-intrinsic consistency we have that $x P^{\prime} y$ implies $\{x, y\} \succ\{y\}$ reaching a contradiction. 


\subsection{Proof of Theorem 4.2}

In order to prove the sufficient part of the Theorem we have to prove that if a reflexive binary relation $\succsim$ is consistent with some asymmetric and transitive binary relation $P$ then $\succsim$ satisfies RI, SC, CC, IC, MR and CR.

- RI: $\{x, y\} \succ\{y\}$ implies, by Definition 2, that $x P y$, which again by the same definition implies $\{x, y\} \sim\{y\}$, and vice versa.

- SC: By the definition of binary relations $P$ and $\otimes$, given any pair $x, y \in X$, either $x P y$, $y P x$ or $x \otimes y$. By Definition 2, if $x P y$ then $\{x, y\} \sim\{x\}$; if $y P x$ then $\{x, y\} \succ\{x\}$; and if $x \otimes y$, then $\{x\} \succ\{x, y\}$.

- CC: By Definition $2\{a\} \succ\{a, x\}$ implies $\neg(a P x)$ and $\neg(x P a)$. Therefore, if $\{a\} \succ$ $\{a, x\} \forall a \in A$ this means that $\nexists a \in A$ s.t $a P x$ or $x P a$, which again by Definition 2 implies $A \succ A \cup\{x\}$.

- IC: Let $x, y \in X$ and $A \in \pi(X)$ such that $y \notin A,\{x, y\} \sim\{x\}$ and $A \cup\{x\} \sim A$. By Definition $2,\{x, y\} \sim\{x\}$ implies $x P y$. If $x \in A$, then, $\exists a \in A(a=x)$ such that $a P y$, and by Definition $2 A \cup\{y\} \sim A$. If $x \notin A$ then, by Definition 2, $A \cup\{x\} \sim A$ implies $\exists a \in A$ such that $a P x$ and, by transitivity of $P, a P y$. Therefore, by Definition 2, $A \cup\{y\} \sim A$.

- MR: Let $A \in \pi(X)$ and $x, y \in X \backslash A$ such that $\neg(\{y\} \sim\{x, y\})$. By Definition 2 $A \cup\{x\} \succ A$ implies $x P a$ for some $a \in \max _{P}(A)$. Also by Definition $2 A \succsim A \cup\{y\}$ implies that, for all $a \in \max _{P}(A), \neg(y P a)$. Therefore $\max _{P}(A) \subseteq \max _{P}(A \cup\{y\})$. This implies that $x P a$ for some $a \in \max _{P}(A \cup\{y\})$, which by Definition 2 implies $A \cup\{x, y\} \succ A \cup\{y\}$.

- CR: Let $A \in \pi(X)$ and $x, y \in X \backslash A$. By Definition $2 A \succ A \cup\{x\}$ implies $x \otimes a$ for all $a \in \max _{P}(A)$. On the other hand, also by Definition 2, $A \cup\{y\} \sim A$ implies $a P y$ for some $a \in A$. Therefore, given that $P$ is transitive, $\max _{P}(A \cup\{y\})=\max _{P}(A)$, which implies $x \otimes a$ for all $a \in \max _{P}(A \cup\{y\})$, and by Definition 2 this implies $A \cup\{y\} \succ A \cup\{x, y\}$.

In order to prove the necessary part of the theorem we have to prove that, if a reflexive binary relation $\succsim$ satisfies all the axioms in Theorem 4.2 , then there exists an asymmetric and transitive binary relation $P$ defined on $X$ with which $\succsim$ is $P$-instrumental consistent. 
We will show that, in particular, $\succsim$ is $P$-consistent with the relation $P$ defined by: $P=\{(x, y)$ such that $\{x, y\} \succ\{y\}\}$. For that purpose:

First, we have to show that $P$ is asymmetric and transitive. Assume $x P y$, then, by the definition of $P,\{x, y\} \succ\{y\}$. Assume that $P$ is not asymmetric and therefore $y P x$. Then $\{x, y\} \succ\{x\}$, but this is in contradiction with RI.

Now we prove the transitivity of $P$ : Take $a, b, c \in X$ such that $a P b$ and $b P c$. By the definition of $P$ this implies $\{a, b\} \sim\{a\}$ and $\{b, c\} \sim\{b\}$. By applying IC (with $a$ in the role of $A ; b$ in the role of $x$ and $c$ in the role of $y$ ), we have $\{a, c\} \sim\{a\}$, this by $R I$ implies $\{a, c\} \succ\{c\}$, which by the definition of $P$ implies $a P c$.

Now, take $A \subseteq \pi(X)$ and $x \in X \backslash A$ :

(i) Let us assume that $x P a$ for some $a \in \max _{P}(A)$ :

Step 1. We claim that $\forall \hat{A} \subset \max _{P}(A), \forall a^{*} \in \max _{P}(A) \backslash \hat{A}, \hat{A} \succ \hat{A} \cup\left\{a^{*}\right\}$. Let $a^{*} \in \hat{A}$. Given that $\hat{a}, a^{*} \in \max _{P}(A)$ we know that $\hat{a} \otimes a^{*}$. This, by the definition of $P$, implies $\neg\left(\left\{\hat{a}, a^{*}\right\} \succ\{\hat{a}\}\right)$ and also $\neg\left(\left\{\hat{a}, a^{*}\right\} \succ\left\{a^{*}\right\}\right)$, which by RI implies $\neg\left(\left\{\hat{a}, a^{*}\right\} \sim\{\hat{a}\}\right)$. Thus we have that $\neg\left(\left\{\hat{a}, a^{*}\right\} \succsim\{\hat{a}\}\right)$, which by SC implies $\{\hat{a}\} \succ\left\{\hat{a}, a^{*}\right\}$. Therefore, by CC, $\hat{A} \succ \hat{A} \cup\left\{a^{*}\right\}$.

Step 2. Let $\max _{P}(A)=\left\{a_{1}^{*}, \ldots, a_{n}^{*}\right\}$. Given that $x P a$ for some $a \in \max _{P}(A)$ there exists $a_{i}^{*} \in \max _{P}(A)$ such that $x P a_{i}^{*}$. Assume, without loss of generality, that $a_{i}^{*}=a_{1}^{*}$. By the definition of $P\left\{x, a_{1}^{*}\right\} \succ\left\{a_{1}^{*}\right\}$.

Step 3. We claim that $\nexists a \in A$ s.t $\{a\} \sim\{x, a\}$. Given that $x P a^{*}$ for some $a^{*} \in \max _{P}(A)$ and given that $P$ is transitive, if there exists some $a \in A$ s.t $a P x$, then $a P a^{*}$ and therefore $a^{*} \notin \max _{P}(A)$, reaching a contradiction. Therefore, $\nexists a \in A$ s.t $a P x$, which by the definition of $P$ and RI implies that $\nexists a \in A$ s.t $\{a\} \sim\{x, a\}$.

Step 4. Now we claim that $\max _{P}(A) \cup\{x\} \succ \max _{P}(A)$. If $\# \max _{P}(A)=1$, then the claim is proved. If $\max _{P}(A)=\left\{a_{1}^{*}, a_{2}^{*}\right\}$ then, given that $a_{1}^{*} \otimes a_{2}^{*}$, as we have shown in Step 1, $\left\{a_{1}^{*}\right\} \succ\left\{a_{1}^{*}, a_{2}^{*}\right\}$. By Step $2\left\{a_{1}^{*}, x\right\} \succ\left\{a_{1}^{*}\right\}$ and by Step $3 \neg\left\{a_{2}^{*}\right\} \sim\left\{a_{2}^{*}, x\right\}$. Thus we can apply MR to obtain $\left\{a_{1}^{*}, a_{2}^{*}, x\right\} \succ\left\{a_{1}^{*}, a_{2}^{*}\right\}$. If $\sharp \max _{P}(A)=2$ Step 4 is proved, if not, By Step $1\left\{a_{1}^{*}, a_{2}^{*}\right\} \succ\left\{a_{1}^{*}, a_{2}^{*}, a_{3}^{*}\right\}$ and by Step 3, $\left\{\left\{a_{3}^{*}\right\} \sim\left\{a_{3}^{*}, x\right\}\right.$, therefore, by MR, $\left\{a_{1}^{*}, a_{2}^{*}, a_{3}^{*}, x\right\} \succ\left\{a_{1}^{*}, a_{2}^{*}, a_{3}^{*}\right\}$. By repeating the same reasoning until exhausting $\max _{P}(A)$ we reach $\max _{P}(A) \cup\{x\} \succ \max _{P}(A)$.

Step 5. We claim that, $\forall A^{\prime} \subseteq A$ s.t $\max _{P}(A) \subseteq\left(A^{\prime}\right) \forall a_{i} \in A \backslash \max _{P}(A), A^{\prime} \sim A^{\prime} \cup\left\{a_{i}\right\}$. If $a_{i} \in A^{\prime}$ then, by reflexivity, $A^{\prime} \sim A^{\prime} \cup\left\{a_{i}\right\}$. If $a_{i} \notin A^{\prime}$ we know that, for all $a_{i} \in$ 
$A \backslash \max _{P}(A)$ there exists $a_{j}^{*} \in \max _{P}(A)$ such that $a_{j}^{*} P a_{i}$. Thus, by the definition of $P$ and RI, $\left\{a_{j}^{*}, a_{i}\right\} \sim\left\{a_{j}^{*}\right\}$. Since $\max _{P}(A) \subseteq\left(A^{\prime}\right)$ we know by reflexivity that $A^{\prime} \sim A^{\prime} \cup\left\{a_{j}^{*}\right\}$. Therefore, by IC, $A^{\prime} \sim A^{\prime} \cup\left\{a_{i}\right\}$.

Step 6. Now, let $A \backslash \max _{P}(A)=\left\{a_{1}, \ldots, a_{m}\right\}$. We know by Step 5 that $\max _{P}(A) \cup$ $\left\{a_{1}\right\} \sim \max _{P}(A)$. By Step 4 we have that $\max _{P}(A) \cup\{x\} \succ \max _{P}(A)$ and by Step 3 $\neg\left\{a_{1}^{*}\right\} \sim\left\{a_{1}^{*}, x\right\}$. MR can be applied to obtain $\max _{P}(A) \cup\left\{a_{1}, x\right\} \succ \max _{P}(A) \cup\left\{a_{1}\right\}$. If $\#\left(A \backslash \max _{P}(A)\right)=1$ the proof is completed. If $\#\left(A \backslash \max _{P}(A)\right)=2$ take $a_{2}$. We have $\max _{P}(A) \cup\left\{a_{1}, x\right\} \succ \max _{P}(A) \cup\left\{a_{1}\right\}$. By Step 3 we have $\neg\left\{a_{2}^{*}\right\} \sim\left\{a_{2}^{*}, x\right\}$ and, given that $\max _{P}(A) \cup\left\{a_{1}\right\} \subset \max _{P}(A)$, we also have that $\max _{P}(A) \cup\left\{a_{1}, a_{2}\right\} \sim \max _{P}(A) \cup\left\{a_{1}\right\}$. Again by MR we obtain $\max _{P}(A) \cup\left\{a_{1}, a_{2}\right\} \cup\{x\} \succ \max _{P}(A) \cup\left\{a_{1}, a_{2}\right\}$, completing the proof. If $\#\left(A \backslash \max _{P}(A)\right)>2$, by repeating the same reasoning until exhausting $A \backslash \max _{P}(A)$ we reach $A \cup\{x\} \succ A$ as desired.

(ii) Now, assume that $a P x$ for some $a \in A$. By the definition of $P\{a, x\} \succ\{x\}$. By RI $\{a, x\} \sim\{a\}$. By reflexivity of $\succsim$ we have $A \cup\{a\} \sim A$ and, given that $\{a, x\} \sim\{a\}$, by IC $A \cup\{x\} \sim A$.

(iii) Now, assume that assumptions (i) and (ii) do not hold, that is, $x \otimes a^{*}$ for all $a^{*} \in \max _{P}(A)$. Then, for all $a^{*} \in \max _{P}(A)$, by the definition of $P$ we have that $\neg\left(\left\{x, a^{*}\right\} \succ\right.$ $\left.\left\{a^{*}\right\}\right)$. We also have that $\neg\left(\left\{x, a^{*}\right\} \succ\{x\}\right)$, which by RI implies $\neg\left(\left\{x, a^{*}\right\} \sim\left\{a^{*}\right\}\right)$. Thus, by SC, $\left\{a^{*}\right\} \succ\left\{x, a^{*}\right\}$ for all $a^{*} \in \max _{P}(A)$. Therefore, by CC, $\max _{P}(A) \succ \max _{P}(A) \cup\{x\}$.

Now, we know that, for all $a \in A \backslash \max _{P}(A)$, there exists $a^{*} \in \max _{P}(A)$ such that $a^{*} P a$ and, by the definition of $P$ and RI, $\left\{a^{*}, a\right\} \sim\left\{a^{*}\right\}$. We can apply the claim of Step 5 in part (i) of the proof to obtain $\max _{P}(A) \cup\{a\} \sim \max _{P}(A)$ and $\forall A^{\prime} \in \pi(X)$ such that $\max _{P}(A) \subseteq A^{\prime} \subset A, \forall a_{i} \in A \backslash A^{\prime}, A^{\prime} \cup\left\{a_{i}\right\} \sim A^{\prime}$.

Thus, let $A \backslash \max _{P}(A)=\left\{a_{1}, \ldots, a_{m}\right\}$. We have already proved that $\max _{P}(A) \succ$ $\max _{P}(A) \cup\{x\}$ and we also have that $\max _{P}(A) \sim \max _{P}(A) \cup\left\{a_{1}\right\}$. By CR this implies $\max _{P}(A) \cup\left\{a_{1}\right\} \succ \max _{P}(A) \cup\left\{a_{1}\right\} \cup\{x\}$. If $\#\left(A \backslash \max _{P}(A)\right)=1$ then the proof is done. If $\#\left(A \backslash \max _{P}(A)\right)=2$ we have that $\max _{P}(A) \cup\left\{a_{1}\right\} \succ \max _{P}(A) \cup\left\{a_{1}\right\} \cup\{x\}$ and that $\max _{P}(A) \cup\left\{a_{1}\right\} \sim \max _{P}(A) \cup\left\{a_{1}\right\} \cup\left\{a_{2}\right\}$. Again by CR we obtain $\max _{P}(A) \cup\left\{a_{1}, a_{2}\right\} \succ$ $\max _{P}(A) \cup\left\{a_{1}, a_{2}, x\right\}$, completing the proof. If $\#\left(A \backslash \max _{P}(A)\right)>2$, then we repeat the same reasoning until exhausting set $A \backslash \max _{P}(A)$ to obtain $A \succ A \cup\{x\}$.

The proof of unicity of $P$ is similar to the corresponding part of the proof for Theorem 4.1 . 


\subsection{Proof of Corollary 3}

It is easy to check that $\succsim$ is $P$-instrumental consistent (i.e. it satisfies the conditions of Definition 1). Next we prove that, if $\succsim$ is transitive and $P$-intrinsic consistent, then the three conditions in the corollary hold:

Let $B \backslash A=\left\{b_{1}, b_{2}, \ldots, b_{k}\right\}$.

(i) Assume, w.l.o.g. that $b_{1} P a$ for all $a \in A$. Given that $\succsim$ is $P$-intrinsic consistent, $A \cup\left\{b_{1}\right\} \succ A$. Given that $\otimes(A)=\otimes(B)$ we know that all the elements in $B \backslash A$ are pairwise related by means of $P$ and that all of them are also related by means of $P$ with the elements of $A$. That is, for any proper subset $B^{\prime}$ of $\left\{b_{2}, \ldots, b_{k}\right\}$, for any $\hat{b} \notin B^{\prime}$, we know by $P$-intrinsic consistency of $\succsim$ that $A \cup\left\{b_{1}\right\} \cup B^{\prime} \cup\{\hat{b}\} \succsim A \cup\left\{b_{1}\right\} \cup B^{\prime}$. Therefore it is possible to construct a chain $\left.B \succsim B \backslash\left\{b_{k}\right\} \succsim B \backslash\left\{b_{k}, b_{k-1}\right\}\right\} \succsim \ldots \succsim A \cup\left\{b_{1}\right\} \succ A$, and by transitivity of $\succsim$ we obtain $B \succ A$.

(ii) By the repeated application of $P$-intrinsic consistency we have $A \succ A \cup\left\{b_{1}\right\} \succ \ldots \succ$ $B$. By transitivity $A \succ B$.

(iii) $\otimes(A)=\otimes(B)$ implies that all the elements in $B \backslash A$ are related by means of $P$ with those of $A$ and also that all the elements in $B \backslash A$ are pairwise related by means of $P$. Given that $\max _{P}(A)=\max _{P}(B)$ and given that by Corollary $1 P$ is acyclic we know that there exists $b^{*} \in B \backslash A$ such that $a P b^{*}$. Let, w.l.o.g. $b^{*}=b_{1}$. Then, by $P$-intrinsic consistency, $A \sim A \cup\left\{b_{1}\right\}$. The argument can be repeated as many times as elements are in $B \backslash A$ to obtain $A \sim A \cup\left\{b_{1}\right\} \sim \ldots \sim B$, and by transitivity of $\succsim, A \sim B$.

We also have to prove that the definition does not enter in contradiction with the assumption of transitivity of $\succcurlyeq$. That is, from the very conditions of Corollary 3 , it is not possible to conclude that, for some $A, B, C \in \pi(X)$ that are pairwise related by set inclusion, $A \succcurlyeq B, B \succcurlyeq C$ and $C \succ A$.

Assume that from the conditions of Corollary 3 we state $A \succcurlyeq B$ and $B \succcurlyeq C$. Note that, if $A \succcurlyeq B$, by either conditions (i), (ii) or (iii), $\otimes(A) \leq \otimes(B)$. Similarly $B \succcurlyeq C$ implies $\otimes(B) \leq \otimes(C)$. Therefore $\otimes(A) \leq \otimes(C)$. Thus, $C \succ A$ is only possible with $\otimes(A)=\otimes(C)$ because none of the conditions of Corollary 3 lead to $C \succ A$ and $\otimes(A)>\otimes(C)$. There are four possible situations under which $A \succcurlyeq B, B \succcurlyeq C$ and $\otimes(A)=\otimes(C)$.

1) $[B \subset A, \otimes(A)=\otimes(B)$ and $\exists a \in A \backslash B$ such that $a P b$ for all $b \in B]$ and $[C \subset$ $B, \otimes(B)=\otimes(C)$ and $\exists b \in B \backslash C$ such that $b P c$ for all $c \in C]$. In this case, given that $C \subset B$ we know by hypothesis that $\exists a \in A \backslash C$ such that $a P c$ for all $c \in C]$ and by the conditions of the corollary $A \succ C$. 
2) $[B \subset A, \otimes(A)=\otimes(B)$ and $\exists a \in A \backslash B$ such that $a P b$ for all $b \in B]$ and $\left[\max _{P}(B)=\right.$ $\max _{P}(C)$ and $\left.\otimes(B)=\otimes(C)\right]$. We distinguish three cases:

- $C \subset B$. Then $C \subset A$ and, by hypothesis, $\exists a \in A \backslash C$ such that $a P c$ for all $c \in C]$. This, by the conditions of the corollary, implies $A \succ C$.

- $B \subset C$ and $C \subset A$. Then $C \succ A$ can only be derived from the conditions in the Corollary if for all $a \in A \backslash C$ there exists $c \in C$ such that $a \otimes c$. This enters in contradiction with $\otimes(A)=\otimes(C)$.

- $B \subset C$ and $A \subset C$. Then, for $C \succ A$ to be derived from the conditions in the Corollary it is necessary that there exists $c \in C \backslash A$ such that $c P a$ for all $a \in A$. Note that any $c \in C$ such that $c P a$ for some $a \in A$ cannot belong to $\max _{P}(C)$ because $\max _{P}(B)=\max _{P}(C)$ and by hypothesis $a P b$ for all $a \in A \backslash B$. Therefore any $c^{*} \in C$ such that $c^{*} P a$ should be such that $c^{*} \notin \max _{P}(C)$. This, given that $P$ is acyclic by Corollary 1 , implies that it is possible to find a chain $c_{1} P c_{2} P \ldots P c^{*}$ such that $c_{1} \in \max _{P}(C)$. We know by hypothesis that $a P c^{\prime}$ for all $c^{\prime} \in \max _{P}(C)$. Therefore we have $a P c_{1} P \ldots P c^{*} P a$, which is in contradiction with the acyclicity of $P$.

3) $\left[\max _{P}(A)=\max _{P}(B)\right.$ and $\left.\otimes(A)=\otimes(B)\right]$ and $[C \subset B, \otimes(B)=\otimes(C)$ and $\exists b \in B \backslash C$ such that $b P c$ for all $c \in C]$.

If $C \subset A, C \succ A$ can only be derived from the conditions in the Corollary if, for all $a \in A \backslash C$, there exists $c \in C$ such that $a \otimes c$. This enters in contradiction with $\otimes(A)=\otimes(C)$.

If $A \subset C$, we reach to a situation that is incompatible with the hypothesis. Note that, given that $\max _{P}(A)=\max _{P}(B), A \subset C$ implies $\max _{P}(B) \subset C$. Therefore it is not possible that, for some $b \in B \backslash C, b P c$ for all $c \in C]$. 4) $\max _{P}(A)=\max _{P}(B)=\max _{P}(C)$ and $\otimes(A)=\otimes(B)=\otimes(C)$. In this case $A \sim C$.

\subsection{Proof of Corollary 4}

Firstly, notice that if $\succsim$ is $P$-instrumental consistent then, by Theorem $4.2, P$ is transititive. Secondly, it is easy to check that $\succsim$ is $P$-instrumental consistent (i.e. it satisfies the conditions of Definition 2). Next we prove that, if $\succsim$ is transitive and $P$-instrumental consistent, then the three conditions in the corollary hold:

Let $B \backslash A=\left\{b_{1}, b_{2}, \ldots, b_{k}\right\}$.

(i) Assume, w.l.o.g. that $\max _{P}(B \backslash A)=\left\{b_{1}\right\}$. We know that there exists $a \in \max _{P}(A)$ s.t $b_{1} P a$, which by Definition 2 implies $A \cup\left\{b_{1}\right\} \succ A$. Now, take $b_{2}$. Given that $b_{2} \notin$ 
$\max _{P}(A)$ and $P$ is transitive we know that $b_{1} P b_{2}$, which again by the same definition implies $A \cup\left\{b_{1}, b_{2}\right\} \sim A \cup\left\{b_{1}\right\}$. Proceeding repeteadly we obtain $A \cup\left\{b_{1}, b_{2}, \ldots, b_{k}\right\} \sim \ldots \sim$ $A \cup\left\{b_{1}, b_{2}\right\} \sim A \cup\left\{b_{1}\right\} \succ A$, which by the transitivity of $\succsim$ implies $B \succ A$.

(ii) Take any $b \in \max _{P}(B \backslash A)$. Since $b$ is incomparable with all the elements in $\max _{P}(A)$, we have by Definition 2 that $\max _{P}(A) \succ \max _{P}(A) \cup\{b\}$. By definition, all the alternatives contained in $\max _{P}(B \backslash A)$ are pairwise incomparable, and by hypothesis all of them are incomparable with the elements in $\max _{P}(A)$. Therefore we can apply repeatedly the definition of $P$-instrumental consistency to obtain $\max _{P}(A) \succ \max _{P}(A) \cup\{b\} \succ$ $\ldots \succ \max _{P}(A) \cup \max _{P}(B)$. Also, by applying repeteadly the definition of $P$-instrumental consistency we know that $\max _{P}(A) \sim A$ and $\max _{P}(B) \sim B$. Thus, by transitivity of $\succsim$, $A \succ B$.

(iii) Assume w.l.o.g. that $A \subset B$. Then, $\max _{P}(A)=\max _{P}(B)$ implies that $\forall b \in B \backslash A$ there exists $a \in A$ s.t $a P b$. Let $B \backslash A=\left\{b_{1}, \ldots, b_{k}\right\}$ and take $b_{1}$. By $P$-instrumental consistency we have that $A \sim A \cup\left\{b_{1}\right\} \sim \ldots \sim B$. By transitivity of $\succsim$ we obtain $A \sim B$.

We also have to prove that the definition does not enter in contradiction with the assumption of transitivity of $\succcurlyeq$.

Assume that from the conditions of Corollary 4 we state $A \succcurlyeq B$ and $B \succcurlyeq C$.

$A \succsim B$ implies either $(1) B \subset A$ and $\max _{P}(A \backslash B)=\left\{a^{*}\right\}$ with $a^{*} P b$ for some $b \in$ $\max _{P}(B) ;(2) A \subset B$ and $a \otimes b \forall a \in \max _{P}(A)$ and $\forall b \in \max _{P}(B \backslash A)$, or $(3) \max _{P}(A)=$ $\max _{P}(B)$.

$B \succsim C$ implies either (4) $C \subset B$ and $\max _{P}(B \backslash C)=\left\{b^{*}\right\}$ with $b^{*} P c$ for some $c \in$ $\max _{P}(C)$; (5) $B \subset C$ and $b \otimes c \forall b \in \max _{P}(B)$ and $\forall c \in \max _{P}(C \backslash B)$, or $(6) \max _{P}(B)=$ $\max _{P}(C)$.

Suppose (1) and (4). Then we have that $a^{*} P b$ for some $b \in \max _{P}(B)$. If $b=b^{*}$, then, by transitivity of $P, a^{*} P c$ for some $c \in \max _{P}(C)$ and the conditions of the corollary imply $A \succ C$. If not, then either $b^{*} \in \max _{P}(B)$ or $b^{*} \notin \max _{P}(B)$. If $b^{*} \in \max _{P}(B)$, then $b^{*}, b \in \max _{P}(B)$. By hypothesis this is only possible if $b \in C$. In this case $a^{*} P c$ for some $c \in \max _{P}(C \backslash A)(c=b)$ and the conditions of the corollary imply $A \succ C$. If $b^{*} \notin \max _{P}(B)$, then, by transitivity of $P, b P b^{*}$, and again by transitivity of $P, a^{*} P b P b^{*} P c$ implies $a^{*} P c$. Then, again by the conditions of the corollary, $A \succ C$.

Suppose (1) and (5). Notice that (5) implies that $\max _{P}(C)=\max _{P}(B) \cup \max _{P}(C)$. The reason is that $b \otimes c \forall b \in \max _{P}(B)$ and $\forall c \in \max _{P}(C \backslash B)$ implies that if $c \in \max _{P}(C \backslash B)$ then $c \in \max _{P}(C)$. Otherwise $\exists b \in B$ such that $b P c$, which by hypothesis is only possible if 
$b \notin \max _{P}(B)$. Therefore, by transitivity of $P, \exists b^{*} \in B$ such that $b^{*} P b P c$, which implies, by transitivity of $P, b^{*} P c$, which is in contradiction with the hypothesis $(5)$. Thus, $\max _{P}(C)=$ $\max _{P}(B) \cup \max _{P}(C)$. Therefore, by hypothesis, $a^{*} P b$ for some $b \in \max _{P}(C)$, which by the conditions of the corollary implies $A \succ C$.

Suppose (1) and (6). Since $\max _{P}(B)=\max _{P}(C)$ we have $a^{*} P b$ for some $b \in \max _{P}(C)$ which by the conditions of the corollary implies $A \succ C$.

Suppose (2) and (4). We distinguish two possibilities. (I) $C \subset A$ and (II) $A \subset C$

- (I) $C \subset A$ implies $C \subset A \subset B$. In this case, for $C \succ A$ to be derived from the conditions in the corollary, it is necessary that $\left[a \otimes c \forall c \in \max _{P}(A \backslash C)\right.$ and $\left.\forall a \in \max _{P}(A)\right]$. By hypothesis $\exists b^{*} \in \max _{P}(B \backslash C)$ such that $b^{*} P c$ for some $c \in \max _{P}(C)$. Notice that $(A \backslash C) \subset(B \backslash C)$. If $b^{*} \in \max _{P}(A \backslash C)$ then $C \succ A$ cannot be derived from the conditions in the corollary. If $b^{*} \notin \max _{P}(A \backslash C)$, then $a^{*} P b^{*}$ for some $a^{*} \in \max _{P}(A \backslash C)$ and we have $a^{*} P b^{*} P c$. Therefore, by transitivity, $\exists a^{*} \in \max _{P}(A \backslash C)$ such that $a^{*} P c$ and, again, $C \succ A$ cannot be derived from the conditions in the corollary.

- (II) $A \subset C$ implies $A \subset C \subset B$. In this case, for $C \succ A$ to be derived from the conditions in the corollary, it is necessary that $\max _{P}(C \backslash A)=\left\{c^{*}\right\}$ and $c^{*} P a$ for some $a \in \max _{P}(A)$. By hypothesis we know that $\exists b^{*} \in \max _{P}(B \backslash C)$ such that $b^{*} P c$ for some $c \in \max _{P}(C)$. Notice that if $c \in A$, given that $A \subset C, c \in \max _{P}(A)$ and then we reach a contradiction with the hypothesis. Therefore $c \in C \backslash A . \quad c \in \max _{P}(C) \operatorname{implies}$ $c \in \max _{P}(C \backslash A)$, and given that $\# \max _{P}(C \backslash A)=1, c=c^{*}$. Therefore we have $b^{*} P c^{*} P a$, which by transitivity implies $b^{*} P a$, reaching again a contradiction. Therefore $C \succ A$ cannot be derived from the conditions in the corollary.

Suppose (2) and (5). By (2) we know that $\max _{P}(B)=\max _{P}(A) \cup \max _{P}(B)$. Therefore, $b \otimes c \forall b \in \max _{P}(B)$ and $\forall c \in \max _{P}(C \backslash B)$ implies $a \otimes c \forall a \in \max _{P}(A)$ and $\forall c \in \max _{P}(C \backslash A)$, which by the conditions of the corollary implies $A \succ C$.

Suppose (2) and (6). The conditions of the corollary lead to $C \succ A$ under two situations: (I) $A \subset C$ and $\max _{P}(C \backslash A) P a$ for some $a \in \max _{P}(A)$ and (II) $C \subset A$ and $[a \otimes c \forall a \in$ $\left.\max _{P}(A \backslash C) \forall c \in \max _{P}(C)\right]$. We prove that both cases lead to a contradiction.

- (I) Let $c^{*}=\max _{P}(C \backslash A)$. Notice that if $A \subset C$ and $c^{*} P a$ for some $a \in \max _{P}(A)$ then $c^{*} \in \max (C)$. Otherwise either $\exists c^{\prime} \in C \backslash A$ such that $c^{\prime} P c^{*}$, in which case $c^{*} \notin \max _{P}(C \backslash A)$ or $\exists c^{\prime} \in A$ such that $c^{\prime} P c^{*}$, in which case $c^{\prime} P c^{*} P a$ and, by transitivity of $P, c^{\prime} P a$, which is in contradiction with $a \in \max _{P}(A)$. Given that $\max _{P}(B)=\max _{P}(C)$ we have that $\exists c^{*} \in$ 
$\max _{P}(B)$ such that $c^{*} P a$, which is only possible if $c^{*} \in B \backslash A$, but this is in contradiction with the hypothesis (2) that $a \otimes b \forall a \in \max _{P}(A)$ and $\forall b \in \max _{P}(B \backslash A)$.

- (II) In this case $C \subset A \subset B$. Given that $\max _{P}(B)=\max _{P}(C)$ we have that $\forall b \in B \backslash C$ there exists $c$ such that $c P b$. Therefore, given that $C \subset A, \forall b \in B \backslash A$ there exists $a$ such that $a P b$. Notice that $a \notin \max _{P}(A)$, otherwise we reach a contradiction with the hypothesis (2) $\left[a \otimes b \forall a \in \max _{P}(B \backslash A) \forall a \in \max _{P}(A)\right]$. This implies that $\exists a^{*} \in \max _{P}(A)$ such that $a^{*} P a$. By transitivity of $P, a^{*} \mathrm{PaPb}$ impies $a^{*} \mathrm{~Pb}$, in which case we reach again a contradiction with the hypothesis $\left[a \otimes b \forall a \in \max _{P}(B \backslash A) \forall a \in \max _{P}(A)\right]$.

Suppose (3) and (4). Let $b^{*}=\max _{P}(B \backslash C)$. If $b^{*} \in \max _{P}(B)$ then, given that $\max _{P}(A)=\max _{P}(B), \exists b^{*} \in \max _{P}(A \backslash C)$ such that $b^{*} P c^{*}$ for some $c^{*} \in \max _{P}(C)$ and, by the conditions of the corollary, $A \succ C$. If $b^{*} \notin \max _{P}(B)$, then $\exists \hat{c} \in C$ such that $\hat{c} P b^{*}$. By transitivity of $P \hat{c} P b^{*} P c^{*}$ implies that $c^{*} \notin \max _{P}(C)$, reaching a contradiction.

Suppose (3) and (5). Then $C \succ A$ can only be derived from the conditions in the corollary if either $\left[A \subset C, \max _{P}(C \backslash A)=c^{*}\right.$ and $c^{*} P a$ for some $\left.a \in \max _{P}(A)\right]$, or $[C \subset A$ and $a \otimes c \forall a \in \max _{P}(A \backslash C)$ and $\left.\forall c \in \max _{P}(C)\right]$.

In the first case, given that $\max _{P}(A)=\max _{P}(B)$, we know that $c^{*} P b$ for some $b \in$ $\max _{P}(B)$. This, by hypothesis, is only possible if $c^{*} \notin \max _{P}(C \backslash B)$. Therefore $\exists \hat{c} \in C \backslash B$ such that $\hat{c} P c^{*}$. By transitivity this implies $\hat{c} P a$ for some $\hat{c} \in C \backslash B$, reaching a contradiction with the hypothesis.

In the second case, we have that $B \subset C \subset A$. Given that $\max _{P}(A)=\max _{P}(B)$, we know that $\forall a \in A \backslash B$ there exists $b \in \max _{P}(B)$ such that $b P a$. Therefore $C \succ A$ in this second case is only possible if $b \notin \max _{P}(C)$, which implies that $\exists c \in C \backslash B$ such that $c P b$. This is again in contradiction with the hypothesis.

Suppose (3) and (6). Then $\max _{P}(A)=\max _{P}(B)=\max _{P}(C)$ which by the conditions in the corollary implies $A \sim B \sim C$.

\subsection{Proof of Theorem 5.1}

First, we prove that if $\succsim$ is consistent with a preference with a structured incompleteness $\left(P, \otimes_{1}, \otimes_{2}\right)$ then $\succsim$ satisfies WRI, SC, IA, MC and CE.

- WRI: Take any $x, y \in X$ such that $\{x, y\} \sim\{y\}$. This, by Definition 3, implies $y P x$, which again by the same definition implies $\{x, y\} \succ\{x\}$. Now, take any $x, y \in X$ such 
that $\{x, y\} \succ\{y\}$. By Definition 3 this implies either $y \otimes_{1} x$ or $x P y$. By the same definition, if $y \otimes_{1} x$ then $\{x, y\} \succ\{x\}$ and if $x P y$ then $\{x, y\} \sim\{x\}$.

- SC: Take any $x, y \in X$ such that $\neg(\{x, y\} \succsim\{x\}) . \neg(\{x, y\} \succ\{x\})$ implies by Definition $3 \neg\left(x \otimes_{1} y\right)$ and $\neg(y P x)$. Moreover, $\neg(\{x, y\} \sim\{x\})$ implies by Definition 3 either $x \otimes_{2} y$ or $\neg(x P y)$. In sum, $\neg(\{x, y\} \succsim\{x\})$ implies necessarily $x \otimes_{2} y$, which by Definition 3 implies $\{x\} \succ\{x, y\}$.

- IA: Let $A \in \pi(X)$ and let $x \in X \backslash A$. Assume $\{a, x\} \succsim\{a\} \forall a \in A$. Thus, $\nexists a \in A$ such that $\{a\} \succ\{a, x\}$, which by Definition 3 implies (i) $\nexists a \in A$ s.t $a \otimes_{2} x$. Assume that there exists at least one element $a^{*} \in A$ such that $\left\{a^{*}, x\right\} \sim\left\{a^{*}\right\}$. This by Definition 3 implies (ii) $a^{*} P x$. Given (i) and (ii), by Definition $3, A \cup\{x\} \sim A$

- MC: Let $A \in \pi(X)$ and let $x \in X \backslash A$. Assume that $\{x, a\} \succ\{a\} \forall a \in A$. This implies, by Definition 3, that, for all $a \in A$, either $x \otimes_{1} a$ or $x P a$. Therefore, by Definition 3, $A \cup\{x\} \succ A$.

- CE: Let $A, B \in \pi(X)$ such that $A \subseteq B$ and let $x \in X \backslash B$. Assume that $A \succ A \cup\{x\}$. by Definition 3 this implies that $\exists a \in A$ such that $x \otimes_{2} a$. Given that $A \subseteq B$, $\exists b \in B,(b=a)$ such that $x \otimes_{2} b$, which by the Definition 3 implies $B \succ B \cup\{x\}$.

In order to prove the necessary part of the theorem, assume that a binary relation $\succsim$ satisfies all the axioms of Theorem 5.1. We have to prove that in this case there exists a preference with a structured incompleteness $\left(P, \otimes_{1}, \otimes_{2}\right)$ defined on $X$ with which $\succsim$ is $\left(P, \otimes_{1}, \otimes_{2}\right)$-intrinsic consistent.

We will show that, in particular, $\succsim$ is consistent with the preference with a structured incompleteness $\left(P, \otimes_{1}, \otimes_{2}\right)$ where $P, \otimes_{1}$ and $\otimes_{2}$ are defined by: $P=\{(x, y)$ such that $\{x, y\} \sim\{x\}\} ; \otimes_{1}=\{(x, y)$ such that $\{x, y\} \succ\{x\}$ and $\{x, y\} \succ\{y\}\}$ and $\otimes_{2}=\{(x, y)$ such that $\{x\} \succ\{x, y\}\}$.

First, we prove that $\left(P, \otimes_{1}, \otimes_{2}\right)$ is a preference with a structured incompleteness:

- $P$ is asymmetric by WRI.

- $\otimes_{1}$ is symmetric by definition.

- $\otimes_{2}$ is symmetric: Assume that $\{x\} \succ\{x, y\}$ but $\neg(\{y\} \succ\{x, y\})$. By SC $\neg(\{y\} \succ$ $\{x, y\})$ implies $\{x, y\} \succsim\{y\}$. By WRI this implies $\{x, y\} \succsim\{x\}$, reaching a contradiction.

- $\otimes_{1} \cap \otimes_{2}=\emptyset$ holds by definition. 
- Now we prove that $X^{2} \backslash P=\otimes_{1} \cup \otimes_{2}: X^{2} \backslash P=\{(x, y) \in X$ such that $\neg(x P y)$ and $\neg(y P x)\} . \neg(x P y)$ and $\neg(y P x)$ implies by definition $\neg(\{x, y\} \sim\{x\})$ and $\neg(\{x, y\} \sim\{y\})$. By SC $\neg(\{x, y\} \sim\{x\})$ implies either $\{x, y\} \succ\{x\}$ or $\{x\} \succ\{x, y\}$. By SC, if $\{x, y\} \succ\{x\}$ there are three possibilities: (i) $\{x, y\} \succ\{y\}$, in which case $x \otimes_{1} y$ by definition; (ii) $\{x, y\} \sim\{y\}$, which is a contradiction with the assumption that $\neg(y P x)$, and (iii) $\{y\} \succ$ $\{x, y\}$, which is in contradiction with WRI. If $\{x\} \succ\{x, y\}$ we have again three possibilities: (i) $\{x, y\} \succ\{y\}$, which is in contradiction with WRI; (ii) $\{x, y\} \sim\{y\}$, which also leads to a contradiction with the assumption that $\neg(x P y)$ and (iii) $\{y\} \succ\{x, y\}$, which by definition implies $x \otimes_{2} y$.

Next we show that, if $\succsim$ satisfies all the axioms in Theorem 5.1 , then it is $\left(P, \otimes_{1}, \otimes_{2}\right)$ consistent with $\left(P, \otimes_{1}, \otimes_{2}\right)$ defined as above:

(i) Assume that for all $a \in A$ either $x P a$ or $x \otimes_{1} a$. Then, either by the definition of $\otimes_{1}$ or by the definition of $P$ and WRI we know that $\{x, a\} \succ\{a\}$, therefore, by MC we have $A \cup\{x\} \succ A$.

(ii) Now, assume that there exists $a$ in $A$ such that $x \otimes_{2} a$. Then, by the definition of $\otimes_{2},\{a\} \succ\{a, x\}$. Since $\{a\} \subseteq A$, by CE, $A \succ A \cup\{x\}$.

(iii) Finally, assume that there exists $a^{*} \in A$ such that $a^{*} P x$ and $\nexists a \in A$ s.t $a \otimes_{2} x$. By the definition of $P$ we have that $\left\{a^{*}, x\right\} \sim\left\{a^{*}\right\}$. Since $\nexists a \in A$ s.t $a \otimes_{2} x$, we have that, for all $a \in A$, either $a P x$, which by $P$ definition implies $\{a, x\} \sim\{a\}$, or $x P a$, or $x \otimes_{1} a$. In these two last cases, as we have shown at (i), we have $\{a, x\} \succ\{a\}$. Therefore $\{a, x\} \succsim\{a\}$ $\forall a \in A$ and $\left\{a^{*}, x\right\} \sim\left\{a^{*}\right\}$, which by IA implies $A \cup\{x\} \sim A$.

\subsection{Proof of Theorem 5.2}

Assume that a reflexive binary relation $\succsim$ satisfies all the axioms of Theorem 5.2. Then there exists a transitive preference with a structured incompleteness $\left(P, \otimes_{1}, \otimes_{2}\right)$ defined on $X$ with which $\succsim$ is $\left(P, \otimes_{1}, \otimes_{2}\right)$-instrumental consistent. In particular, consider $P, \otimes_{1}$ and $\otimes_{2}$ defined as in the proof of Theorem 5.1. That is, $P=\{(x, y)$ such that $\{x, y\} \sim\{x\}\} ; \otimes_{1}=\{(x, y)$ such that $\{x, y\} \succ\{x\}$ and $\{x, y\} \succ\{y\}\}$ and $\otimes_{2}=\{(x, y)$ such that $\{x\} \succ\{x, y\}\}$ . In the proof of Theorem 5.1 it is proved, by using axioms WRI and SC, that $\left(P, \otimes_{1}\right.$, $\left.\otimes_{2}\right)$ is a preference with a structured incompleteness. Given that WRI and SC are also characterization axioms in Theorem 5.2 that part of the proof is identical. It only remains then to prove that $P$ is, in addition, transitive: Take $a, b, c \in X$ such that $a P b$ and $b P c$. By the definition of $P$ this implies $\{a, b\} \sim\{a\}$ and $\{b, c\} \sim\{b\}$. By applying IC (with $a$ 
in the role of $A ; b$ in the role of $x$ and $c$ in the role of $y$ ), we have $\{a, c\} \sim\{a\}$, which by the definition of $P$ implies $a P c$.

Next we have to prove that, if a reflexive binary relation $\succsim$ satisfies all the axioms in Theorem 5.2, then it is $\left(P, \otimes_{1}, \otimes_{2}\right)$-instrumental consistent:

(i) Assume that $\exists a \in(A)$ s.t $a P x$. Then by the definition of $P$ we know that $\{a, x\} \sim$ $\{a\}$. By reflexivity of $\succsim$ we have $A \cup\{a\} \sim A$. Therefore, by IC, $A \cup\{x\} \sim A$.

(ii) Assume now that $\exists a_{i} \in \max _{P}(A)$ s.t $x P a_{i}$ or $x \otimes_{1} a_{i}$; $\nexists a \in \max _{P}(A)$ s.t $a P x$, and $\forall a^{*} \in \max _{P}(A)$ s.t $a^{*} \otimes_{2} x \nexists a \in \max _{P}(A)$ s.t $a \otimes_{1} a^{*}$.

Step 1. Let $\hat{A}=\left\{a \in \max _{P}(A): x P a\right.$ or $\left.x \otimes_{1} a\right\}$. For all $a \in \hat{A}$, if $x P a$, by the definition of $P,\{x, a\} \sim\{x\}$, and by WRI $\{x, a\} \succ\{a\}$. If $x \otimes_{1} a$, by the definition of $\otimes_{1}$, $\{x, a\} \succ\{a\}$. Thus, for all $a \in \hat{A},\{x, a\} \succ\{a\}$. Then, by MC, we have that $\hat{A} \cup\{x\} \succ \hat{A}$.

Step 2. In this step we prove that $\{x\} \succ\{x, \bar{a}\} \forall \bar{a} \in \max _{P}(A) \backslash \hat{A}$. Given that $\bar{a} \in A \backslash \hat{A}$ we know that $\neg(x P \bar{a})$ and $\neg\left(x \otimes_{1} \bar{a}\right)$. By assumption $\nexists a \in \max _{P}(A)$ s.t $a P x$, therefore $x \otimes_{2} \bar{a}$ $\forall \bar{a} \in \max _{P}(A) \backslash \hat{A}$, which by the definition of $\otimes_{2}$ implies $\{x\} \succ\{x, \bar{a}\} \forall \bar{a} \in \max _{P} A \backslash \hat{A}$.

Step 3. Let $\max _{P}(A) \backslash \hat{A}=\left\{\bar{a}_{1}, \ldots, \bar{a}_{n}\right\}$. Take any $\bar{a}_{j} \in \max _{P}(A) \backslash \hat{A}$. Given that $\bar{a}_{j} \in \max _{P}(A)$ we know that $\forall \hat{a} \in \hat{A}$ neither $\bar{a}_{j} P \hat{a}$ nor $\hat{a} P \bar{a}_{j}$. At Step 2 we have proved that $x \otimes_{2} \bar{a}_{j} \forall \bar{a}_{j} \in \max _{P}(A) \backslash \hat{A}$. Recall that, by assumption, $\forall a^{*} \in \max _{P}(A)$ s.t $a^{*} \otimes_{2} x$ $\nexists a \in \max _{P}(A)$ s.t $a \otimes_{1} a^{*}$. Thus, for all $a \in \max _{P}(A), \neg\left(\bar{a}_{j} \otimes_{1} a\right)$, which implies $\bar{a}_{j} \otimes_{2} a$ $\forall a \in \max _{P}(A) \backslash\left\{\bar{a}_{j}\right\}$. Take $\bar{a}_{1}$. By CC we know that $\hat{A} \succ \hat{A} \cup\left\{\bar{a}_{1}\right\}$; by Step $1 \hat{A} \cup\{x\} \succ \hat{A}$, and by Step $3\{x\} \succ\left\{x, \bar{a}_{1}\right\}$. Therefore, we can apply MR to obtain $\hat{A} \cup\left\{x, \bar{a}_{1}\right\} \succ \hat{A} \cup\left\{\bar{a}_{1}\right\}$. If $\max _{P}(A) \backslash \hat{A}=\left\{\bar{a}_{1}\right\}$ we have $\max _{P}(A) \cup\{x\} \succ \max _{P}(A)$. If not, take $\left\{\bar{a}_{2}\right\}$. We have $\hat{A} \cup\left\{x, \bar{a}_{1}\right\} \succ \hat{A} \cup\left\{\bar{a}_{1}\right\}$; by CC $\hat{A} \cup\left\{\bar{a}_{1}\right\} \succ \hat{A} \cup\left\{\bar{a}_{1}, \bar{a}_{2}\right\}$, and by Step $2\{x\} \succ\left\{x, \bar{a}_{2}\right\}$. Thus, we can apply MR to obtain $\hat{A} \cup\left\{x, \bar{a}_{1}, \bar{a}_{2}\right\} \succ \hat{A} \cup\left\{\bar{a}_{1}, \bar{a}_{2}\right\}$. Proceeding similarly until exhausting all elements of $\max _{P}(A) \backslash \hat{A}$ we reach $\max _{P}(A) \cup\{x\} \succ \max _{P}(A)$.

Step 4. Let $A \backslash \max _{P}(A)=\left\{a_{1}, \ldots, a_{n}\right\}$. Take $a_{1}$. We know that there exists $a^{*} \in$ $\max _{P}(A)$ such that $a^{*} P a_{1}$. Thus, by the definition of $P,\left\{a^{*}, a_{1}\right\} \sim\left\{a^{*}\right\}$. By reflexivity of $\succsim$, $\max _{P}(A) \cup\left\{a^{*}\right\} \sim \max _{P}(A)$. Thus, we can apply IC to obtain $\max _{P}(A) \sim \max _{P}(A) \cup\left\{a_{1}\right\}$. By hypothesis $\nexists a \in \max _{P}(A)$ such that $a P x$, thus, by transitivity of $P$, $\nexists a \in A$ such that $a P x$, and by the definition of $P, \neg\left(\left\{a_{1}\right\} \sim\left\{x, a_{1}\right\}\right)$. By Step $3 \max _{P}(A) \cup\{x\} \succ$ $\max _{P}(A)$. Thus, we can apply MR to obtain $\max _{P}(A) \cup\left\{x, a_{1}\right\} \succ \max _{P}(A) \cup\left\{a_{1}\right\}$. If $A \backslash \max _{P}(A)=\left\{a_{1}\right\}$ Step 4 is proved. If not take $a_{2} \in A \backslash \max _{P}(A)=\left\{a_{1}, \ldots, a_{n}\right\}$. Again, given that $a_{2} \notin \max _{P}(A)$ there exists $a^{*^{\prime}} \in \max _{P}(A) \cup\left\{a_{1}\right\}$ such that $a^{*^{\prime}} P a_{2}$ and by applying similarly the definition of $P$, reflexivity of $\succsim$ and IC we obtain $\max _{P}(A) \cup\left\{a_{1}\right\} \sim$ 
$\max _{P}(A) \cup\left\{a_{1}, a_{2}\right\}$. Again, by hypothesis, $\nexists a \in \max _{P}(A)$ such that $a P x$, and by transitivity of $P$ and the definition of $P$ we obtain $\neg\left(\left\{a_{2}\right\} \sim\left\{x, a_{2}\right\}\right)$. Then, given that $\max _{P}(A) \cup$ $\left\{x, a_{1}\right\} \succ \max _{P}(A) \cup\left\{a_{1}\right\}$, by MR we have $\max _{P}(A) \cup\left\{x, a_{1}, a_{2}\right\} \succ \max _{P}(A) \cup\left\{a_{1}, a_{2}\right\}$. Proceeding similarly until exhausting all elements of $A$ we finally obtain $A \cup\{x\} \succ A$.

(iii) Now, assume that $x \otimes_{2} a^{*}$ for all $a^{*} \in \max _{P}(A)$. By CC $\max _{P}(A) \succ \max _{P}(A) \cup\{x\}$. We can apply the definition of $P$, reflexivity of $\succsim$ and IC like in Step 4 above to prove that for all $a \in A \backslash \max _{P}(A), \max _{P}(A) \sim \max _{P}(A) \cup\{a\}$. Thus, let $A \backslash \max _{P}(A)=\left\{a_{1}, \ldots, a_{k}\right\}$. We have that $\max _{P}(A) \succ \max _{P}(A) \cup\{x\}$ and $\max _{P}(A) \sim \max _{P}(A) \cup\left\{a_{1}\right\}$. By CR this implies $\max _{P}(A) \cup\left\{a_{1}\right\} \succ \max _{P}(A) \cup\left\{a_{1}\right\} \cup\{x\}$. If $A \backslash \max _{P}(A)=\left\{a_{1}\right\}$ then the proof is done. If $A \backslash \max _{P}(A)=\left\{a_{1}, a_{2}\right\}$ we have that $\max _{P}(A) \cup\left\{a_{1}\right\} \succ \max _{P}(A) \cup\left\{a_{1}\right\} \cup\{x\}$ and that $\max _{P}(A) \cup\left\{a_{1}\right\} \sim \max _{P}(A) \cup\left\{a_{1}\right\} \cup\left\{a_{2}\right\}$. Again by CR we obtain $\max _{P}(A) \cup\left\{a_{1}, a_{2}\right\} \succ$ $\max _{P}(A) \cup\left\{a_{1}, a_{2}, x\right\}$, completing the proof. If $\#\left(A \backslash \max _{P}(A)\right)>2$, then we repeat the same reasoning until exhausting the set $A \backslash \max _{P}(A)$ to obtain $A \succ A \cup\{x\}$.

\subsection{Proof of Theorem 5.3}

- WRI: Take any $x, y \in X$ such that $\{x, y\} \sim\{y\}$. This, by Definition 4, implies $y P x$, which again by the same definition implies $\{x, y\} \succ\{x\}$. Now, take any $x, y \in X$ such that $\{x, y\} \succ\{y\}$. By Definition 4 this implies either $x P y$ or $x \otimes_{1} y$. By Definition 4 , if $y \otimes_{1} x$, then $\{x, y\} \succ\{x\}$ and if $x P y$, then $\{x, y\} \sim\{x\}$.

- SC: Take any $x, y \in X$ such that $\neg(\{x, y\} \succsim\{x\}) . \neg(\{x, y\} \succ\{x\})$ implies by Definition $4 \neg\left(x \otimes_{1} y\right)$ and $\neg(y P x)$. Moreover, $\neg(\{x, y\} \sim\{x\})$ implies by Definition 4 $\neg(x P y)$. Therefore, by the properties assumed on $\left(P, \otimes_{1}, \otimes_{2}\right), \neg(\{x, y\} \succsim\{x\})$ implies necessarily $x \otimes_{2} y$, which by Definition 4 implies $\{x\} \succ\{x, y\}$.

- CC: Let $\{a\} \succ\{a, x\} \forall a \in A$. This, by Definition 4, implies $x \otimes_{2} a \forall a \in A$, therefore $x \otimes_{2} a \forall a \in \max _{P}(A)$, which by Definition 4 implies $A \succ A \cup\{x\}$.

- IC: Let $x, y \in X$ and $A \in \pi(X)$ such that $x \notin A,\{x, y\} \sim\{x\}$ and $A \cup\{x\} \sim A$. By Definition $4\{x, y\} \sim\{x\}$ implies $x P y$. We distinguish two possibilities: either $y \in A$ or $y \notin A$. If $y \in A$ then $A \cup\{y\} \sim A$ holds by reflexivity of $\succsim$. If $y \notin A$ then, by Definition $4, \exists a \in A$ such that $a P x$. By the transitivity of $P, a P y$. Thus, by Definition $4, A \cup\{y\} \sim A$.

- MR: Let $A \in \pi(X)$ and let $x, y \in X \backslash A$. Assume that $A \cup\{x\} \succ A ; A \succsim A \cup\{y\}$ and $\neg(\{y\} \sim\{x, y\})$. 
$A \cup\{x\} \succ A$ implies by Definition 4 that $\exists a_{i} \in \max _{P}(A)$ such that either $x P a_{i}$ or $a_{i} \otimes_{1} x ; \nexists a \in \max _{P}(A)$ s.t $a P x$, and $\left.\forall a^{*} \in \max _{P}(A)\right)$ s.t $x \otimes_{2} a^{*} \nexists a \in \max _{P}(A)$ s.t $a \otimes_{1} a^{*}$.

Assume first that $A \sim A \cup\{y\}$. This implies, by Definition 4, that $\exists a \in A$ s.t $a P y$. Thus, given the transitivity of $P, \max _{P}(A)=\max _{P}(A \cup\{y\})$. Therefore: (i) $\exists a \in \max _{P}(A \cup\{y\})$ such that $x P a_{i}$ or $a_{i} \otimes_{1} x$ and (ii) $\forall a^{*} \in \max _{P}(A \cup\{y\})$ s.t $x \otimes_{2} a^{*}$ $\nexists a \in \max _{P}(A \cup\{y\})$ s.t $a \otimes_{1} a^{*}$ and (iii) $\nexists a \in A \cup\{y\}$ s.t $a P x$. That is, according to Definition 4 all the conditions ((i), (ii) and (iii)) for $A \cup\{x, y\} \succ A \cup\{y\}$ hold.

Now, assume that $A \succ A \cup\{y\}$. By Definition 4 this implies $y \otimes_{2} a \forall a \in \max _{P}(A)$. Therefore $\max _{P}(A \cup\{y\})=\max _{P}(A) \cup\{y\}$, which implies (i) $\exists a \in \max _{P}(A \cup\{y\})$ such that $x P a_{i}$ or $a_{i} \otimes_{1} x . \neg(\{y\} \sim\{x, y\})$ implies $\neg(y P x)$, therefore, given that $\nexists a \in \max _{P}(A)$ s.t $a P x$, we have that (ii) $\nexists a \in \max (A \cup\{y\})$ s.t $a P x$. Given that $\neg(y P x)$, we distinguish three possibilities: If it is the case that $x \otimes_{2} y$, since $y \otimes_{2} a \forall a \in$ $\max _{P}(A)$, we know that $\nexists a \in \max _{P}(A)$ s.t $a \otimes_{1} y$. We also know that $\forall a^{*} \in \max _{P}(A)$ s.t $x \otimes_{2} a^{*} \nexists a \in \max _{P}(A)$ s.t $a \otimes_{1} a^{*}$. Therefore, (iii) $\forall a^{*} \in \max _{P}(A \cup\{y\})$ s.t $x \otimes_{2} a^{*}$ $\nexists a \in \max _{P}(A \cup\{y\})$ s.t $a \otimes_{1} a^{*}$. Thus, the three conditions for $A \cup\{x, y\} \succ A \cup\{y\}$ hold. If it is the case that either $x \otimes_{1} y$ or $x P y$, we already know that $\forall a^{*} \in \max _{P}(A)$ s.t $x \otimes_{2} a^{*} \nexists a \in \max _{P}(A)$ s.t $a \otimes_{1} a^{*}$. Given that $\neg\left(x \otimes_{2} y\right)$, we have that (iii) $\forall a^{*} \in \max _{P}(A \cup\{y\})$ s.t $\left.x \otimes_{2} a^{*} \nexists a \in \max _{P}(A \cup\{y\})\right)$ s.t $a \otimes_{1} a^{*}$. Thus, again, the three conditions for $A \cup\{x, y\} \succ A \cup\{y\}$ hold.

- MC: Assume that $\forall A \in \pi(X), \forall x \in X \backslash A,\{x, a\} \succ\{a\} \forall a \in A$. Then, by Definition 4, either $x P a$ or $x \otimes_{1} a \forall a \in A$. Therefore, by Definition $4, A \cup\{x\} \succ A$.

- CR: Let $A \in \pi(X)$ and $x, y \in X \backslash A$. By Definition $4, A \succ A \cup\{x\}$ implies $x \otimes_{2} a$ for all $a \in \max _{P}(A)$. On the other hand, also by Definition $4, A \cup\{y\} \sim A$ implies $a P y$ for some $a \in A$. Therefore, given the transitivity of $P, \max _{P}(A \cup\{y\})=\max _{P}(A)$, which implies that $x \otimes_{2} a$ for all $a \in \max _{P}(A \cup\{y\})$, and by Definition 4 this implies $A \cup\{y\} \succ A \cup\{x, y\}$.

\section{References}

Arlegi, R. and Nieto, J. (2001a). Incomplete preferences and the preference for flexibility. Math Soc Sci, 41(2):151-165. 
Arlegi, R. and Nieto, J. (2001b). Ranking opportunity sets: An approach based on the preference for flexibility. Soc Choice Welf, 18(1):23-36.

Barberà, S., Bossert, W., and Pattanaik, P. K. (2004). Ranking sets of objects. In Handbook of utility theory, pages 893-977. Springer.

Bossert, W., Pattanaik, P. K., and Xu, Y. (1994). Ranking opportunity sets: an axiomatic approach. J Econ Theory, 63(2):326-345.

Danan, E., Guerdjikova, A., and Zimper, A. (2012). Indecisiveness aversion and preference for commitment. Theor Decis, 72(1):1-13.

Danan, E. and Ziegelmeyer, A. (2006). Are Preferences Complete?: An Experimental Measurement of Indecisiveness Under Risk. Max Planck Inst. of Economics, Strategic Interaction Group.

Dekel, E., Lipman, B. L., Rustichini, A., and Sarver, T. (2007). Representing preferences with a unique subjective state space: A corrigendum. Econometrica, 75(2):591-600.

Eliaz, K. and Ok, E. A. (2006). Indifference or indecisiveness? Choice-theoretic foundations of incomplete preferences. Game Econ Behav, 56(1):61-86.

Gerasimou, G. (2017). Indecisiveness, undesirability and overload revealed through rational choice deferral. The Econ J, 128(614):2450-2479.

Guerdjikova, A. and Zimper, A. (2008). Flexibility of choice versus reduction of ambiguity. Soc Choice Welf, 30(3):507-526.

Gul, F. and Pesendorfer, W. (2001). Temptation and Self-Control. Econometrica, 69(6):1403-1435.

Iyengar, S. S. and Lepper, M. R. (2000). When choice is demotivating. J Pers Soc Psychol, 79(6):995-1006.

Kreps, D. M. (1979). A Representation Theorem for "Preference for Flexibility". Econometrica, 47(3):565-577.

May, K. O. (1954). Intransitivity, utility, and the aggregation of preference patterns. Econometrica: Journal of the Econometric Society, pages 1-13.

Nehring, K. (1999). Preference for flexibility in a savage framework. Econometrica, 67(1):101-119. 
Ortoleva, P. (2013). The price of flexibility: Towards a theory of Thinking Aversion. $J$ Econ Theory Theory, 148(3):903-934.

Pattanaik, P. K. and Xu, Y. (1990). On ranking opportunity sets in terms of freedom of choice. Rech Econ Louvain, 56(3-4):383-390.

Pejsachowicz, L. and Toussaert, S. (2017). Choice deferral, indecisiveness and preference for flexibility. Journal of Economic Theory, 170:417-425.

Puppe, C. (1996). An axiomatic approach to preference for freedom of choice. J Econ Theory, 68(1):174-199.

Sarver, T. (2008). Anticipating regret: Why fewer options may be better. Econometrica, $76(2): 263-305$.

Schwartz, B., Ward, A., Lyubomirsky, S., Monterosso, J., White, K., and Lehman, D. R. (2002). Maximizing versus satisficing: Happiness is a matter of choice. J Pers Soc Psychol, 83(5):1178-1197.

Sen, A. (1988). Freedom of choice: concept and content. Eur Econ Rev, 32(2-3):269-294.

Sen, A. (1997). Maximization and the Act of Choice. Econometrica, 65(4):745.

Sonsino, D. and Mandelbaum, M. (2001). On preference for flexibility and complexity aversion: Experimental evidence. Theor Decis, 51(2-4):197-216.

Styron, W. (1979). Sophie's choice. Random House.

Tversky, A. (1969). Intransitivity of preferences. Psychological review, 76(1):31. 